\title{
Mobile M2M communication architectures, upcoming challenges, applications, and future directions
}

Yasir Mehmood ${ }^{1 *}$, Carmelita Görg ${ }^{1}$, Maciej Muehleisen ${ }^{2}$ and Andreas Timm-Giel ${ }^{2}$

\begin{abstract}
Machine-to-machine (M2M) communication is becoming an increasingly important part of mobile traffic and thus also a topic of major interest for mobile communication research and telecommunication standardization bodies. M2M communication offers various ubiquitous services and is one of the main enablers of the vision inspired by the Internet of Things (loT). The concept of mobile M2M communication has emerged due to the wide range, coverage provisioning, high reliability, and decreasing costs of future mobile networks. Nevertheless, M2M communications pose significant challenges to mobile networks, e.g., due to the expected large number of devices with simultaneous access for sending small-sized data, and a diverse application range. This paper provides a detailed survey of M2M communications in the context of mobile networks, and thus focuses on the latest Long-Term Evolution-Advanced (LTE-A) networks. Moreover, the end-to-end network architectures and reference models for M2M communication are presented. Furthermore, a comprehensive survey is given to M2M service requirements, major current standardization efforts, and upcoming M2M-related challenges. In addition, an overview of upcoming M2M services expected in 5G networks is presented. In the end, various mobile M2M applications are discussed followed by open research questions and directions.
\end{abstract}

Keywords: Machine-to-machine, Internet of Things, End-to-end, Long-term evolution-advanced, Standardization efforts, $5 G$

\section{Review}

\subsection{Introduction}

In recent years, collaborative communication between numerous intelligent systems through either mobile or fixed networks has achieved significant importance. One of the resultant emerging domains is mobile machineto-machine (M2M) communication. M2M communication describes a communication style in which two or more entities such as devices/machines communicate with each other autonomously [1]. M2M communication is playing a promising role in enabling the Internet of Things (IoT) vision by providing ubiquitous connectivity between numerous intelligent devices [2]. The term $\mathrm{M} 2 \mathrm{M}$ in IoT describes the autonomous exchange of information among numerous devices inter-connected with each other. M2M communication thus covers a wide

\footnotetext{
*Correspondence: ym@comnets.uni-bremen.de

${ }^{1}$ Communication Networks, University of Bremen, Bremen, Germany

Full list of author information is available at the end of the article
}

range of use cases [3-5]. For instance, the M2M application area includes intelligent transportation systems (ITS), logistics and supply chain management, smart metering, e-healthcare, surveillance and security, smart cities, and home automation. Particularly, many intelligent devices are expected to be deployed in the automotive sector, security, e-healthcare, and logistics [6]. Consequently, $\mathrm{M} 2 \mathrm{M}$ communications are anticipated to reshape the business/revenue of telecom operators, M2M enterprises, and M2M enablers due to the emerging use of intelligent sensors and actuators in many advanced aforementioned applications [7].

Mobile standards are foreseen to play a prominent role in the successful deployment of numerous M2M applications due to several reasons, e.g., high capacity to support a large number of devices, enhanced coverage, low power consumption, high reliability, low cost modules, as well as easy and short-term deployment opportunities [2]. Several forecasts have reported a considerable 
market growth for M2M device volume. Some of these recent forecasts predicting the number of cellular-based M2M devices worldwide are shown in Fig. 1. Therefore, M2M services are considered as one of the main motivations behind the tremendous growth in mobile traffic [6]. Furthermore, mobile networks such as Long-Term Evolution/Long-Term Evolution-Advanced (LTE/LTE-A) offer high data rates, enhanced coverage, and improved radio resource management mechanisms. However, the cellular systems are particularly designed to support the exponentially growing human-to-human/human-tomachine $(\mathrm{H} 2 \mathrm{H} / \mathrm{H} 2 \mathrm{M})$ traffic by enabling improved broadband services [8]. For instance, Ericsson in its mobility report [9] anticipates approximately 5.6 billion cellularbased active smartphones by 2019. In order to support increasing mobile traffic, several enhancements in existing LTE systems [10] such as femtocells [11] and relay nodes (RNs) [12] were introduced to fulfill the International Mobile Telecommunications-Advanced (IMTAdvanced) demands of maximum throughput of up to $1 \mathrm{Gbps}$ in downlink and $500 \mathrm{Mbps}$ in uplink [13].

Several review papers [14-22] discussed M2M communications in the context of wireless technologies by focusing on various M2M applications such as ehealthcare, smart metering, smart grids, IoT paradigm, and radio resource management. For instance, authors in [14] addressed the M2M e-healthcare application, i.e., $\mathrm{m}$-Health, by highlighting the benefits of using M2M technology for both patients and service providers due to reliable autonomous communication. Furthermore, in [14], a first detailed analysis of wireless body area networks (WBANs) towards the patient side is provided, and various end-to-end (E2E) solutions for practical e-healthcare applications were discussed. In [20], the authors surveyed a number of existing communication technologies which can be used for M2M communication in smart grids. Furthermore, the authors presented the most suitable technologies such as Institute of Electrical and Electronics Engineering (IEEE) 802.15.3a (Ultra Wide Band), IEEE 802.15.1 (Bluetooth), and IEEE 802.15.4 (ZigBee) for smart grids by addressing their limitations as well. Authors in [22] surveyed existing Internet Engineering Task Force (IETF) protocols for supporting the IoT paradigm. In [23], authors discussed applicability of software-defined networks and network virtualization to mobile and wireless networks. Additionally, authors in [15] highlighted the correlation between M2M, wireless sensors networks (WSNs), cyber-physical systems (CPS), and the IoT. Furthermore, authors in [15] proposed an M2M system integrating intelligent roads with unmanned vehicles. In [16], authors surveyed several M2M communication scenarios in the context of wireless technology to cloud and a machine swarm of a large number of devices and explored their practical realizations. In $[17,18]$, authors presented various radio resource management and scheduling schemes for mobile M2M communications. Moreover, issues regarding random access due to the large number of devices using mobile networks are addressed, e.g., in [19] and [21].

To the best of the authors' knowledge, a comprehensive survey covering existing mobile M2M communication architectures, service requirements, existing and upcoming challenges, up-to-date standardization efforts, application scenarios, upcoming M2M services in 5G networks, as well as future directions is not available. Therefore, the objective of this survey is to give a comprehensive overview on the aforementioned topics which helps the reader to understand the concept, future trends, and requirements of mobile M2M communication. Since LTE and LTE-A are the latest Third Generation Partnership Project (3GPP) mobile standards, the rest of the paper focuses on M2M communications in LTE/LTE-A

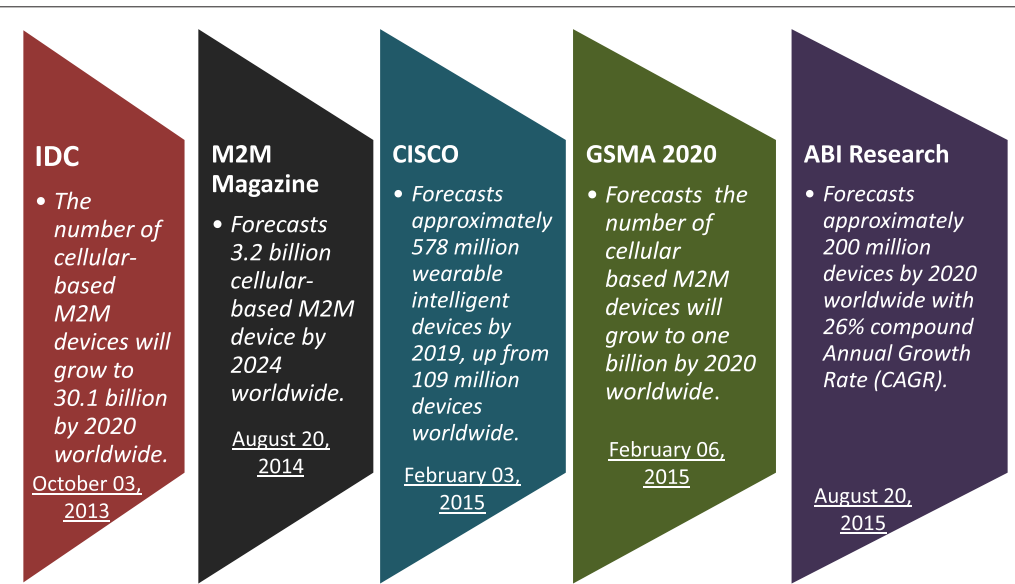

Fig. 1 Recent forecasts about cellular-based M2M traffic volume by IDC [141], M2M magazine [88], CISCO [85], GSMA [87], and ABI research [6] 
networks. Furthermore, an overview of the rest of the survey and a list of most frequently used acronyms is presented in Fig. 2 and Table 1, respectively.

\subsection{M2M, loT, and CPS: an overview}

The use of communication technologies for ubiquitous connectivity among numerous devices/machines has achieved significant importance. As a result, notable developments have been made in several domains such as M2M, WSNs, IoT, and CPS. Since the key idea in the above domains is identical, the authors discussed correlations among M2M, WSNs, IoT, and CPS in [24]. M2M communication offers autonomous communication between intelligent sensors and actuators. Therefore, M2M traffic characteristics differ from conventional mobile traffic, e.g., in terms of traffic density, packet size, and quality of service (QoS) requirements [25]. For instance, Fig. 3 presents some of the data sizes of various M2M uses cases. Additionally, an experimental study done in [26] shows that M2M traffic exhibits a significantly different behavior than the traditional smartphone (mobile) traffic in various aspects. For example, unlike traditional mobile traffic which is dominant in downlink rather than in uplink, M2M is an uplink-dominant traffic which particularly generates bursty traffic volumes. Furthermore, M2M devices compete with smartphones for network resources in co-geographical regions. Thus, supporting a large number of devices and issues such as spectral efficiency enhancements in the presence of M2M traffic, latency control, and limitation of network congestion demands utmost attention. These findings suggest that more careful spectrum allocation and utilization schemes are needed, so that M2M communications can benefit from mobile networks such as LTE/LTE-A without degrading the performance of their traditional traffic. For these reasons, well-known standardization bodies such as 3GPP and European Telecommunication Standardization Institute (ETSI) have already started putting efforts to support a large number of devices while considering their characteristics and service requirements. In addition, 3GPP and ETSI have already proposed an E2E reference model and functional architectures, respectively $[27,28]$. Moreover, 3GPP has considered to investigate $\mathrm{MTC}^{1}$ service requirements and subsequently proposed various schemes for system improvements in the presence of MTC.

The term IoT describes connecting objects/machines often called things with an infrastructure based on the Internet. The fundamental components of IoT include sensing/acquiring data, heterogeneous connectivity, data processing, as well as applications and services. Additionally, security and privacy are also considered as essential components in IoT. Moreover, in IoT, WSNs are usually considered as a fundamental scenario which spatially distributes autonomous sensors for monitoring various physical and environmental factors, thus to deliver the acquired information to backend stations. IoT covers a vast application area such as greenhouse monitoring, ITS,

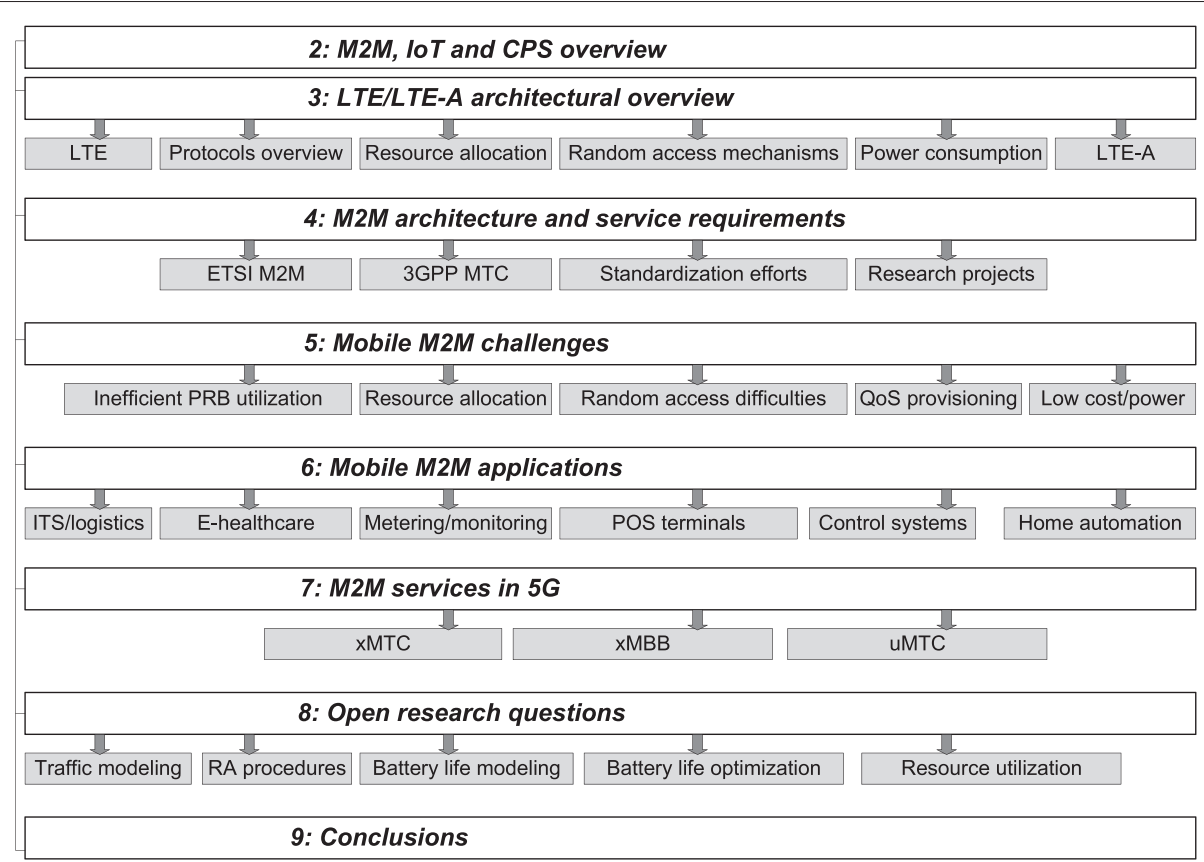

Fig. 2 An overview of the survey 
Table 1 List of used abbreviations

\begin{tabular}{|c|c|}
\hline Abbreviations & Acronyms \\
\hline AAA & Accounting, authorization, and authentication \\
\hline $\mathrm{ARQ}$ & Automatic repeat request \\
\hline AS & Access stratum \\
\hline$C P$ & Cyclic prefix \\
\hline CMPP & Coupled Markov modulated Poisson process \\
\hline \multirow[t]{2}{*}{ IMT-Advanced } & International Mobile Telecommunications- \\
\hline & Advanced \\
\hline EPS & Evolved packet system \\
\hline E-UTRAN & Evolved UMTS terrestrial radio network \\
\hline GERAN & GSM EDGE radio access network \\
\hline GGSN & Gateway GPRS support node \\
\hline GSM & Global system for mobile communications \\
\hline HARQ & Hybrid automatic repeat request \\
\hline HLR & Home location register \\
\hline HSDPA & High-speed downlink packet access \\
\hline HSS & Home subscriber server \\
\hline HSUPA & High-speed uplink packet access \\
\hline $\mathrm{ICT}$ & Information and communication technology \\
\hline ITU & International Telecommunication Union \\
\hline MAC & Medium access control \\
\hline MME & Mobility Management Entity \\
\hline MMPP & Markov modulated Poisson process \\
\hline MSC & Mobile switching center \\
\hline MTC-IWF & MTC InterWorking Function \\
\hline NAS & Non access stratum \\
\hline OMA & Open mobile alliance \\
\hline PDNs & Packet data networks \\
\hline P-GW & Packet gateway \\
\hline $\mathrm{PHY}$ & Physical layer \\
\hline PLMN & Public land mobile network \\
\hline PSM & Power saving mode \\
\hline PRB & Physical resource block \\
\hline QCl & QoS class identifiers \\
\hline $\mathrm{RLC}$ & Radio link control \\
\hline RRC & Radio resource control \\
\hline SAE & System architecture evolution \\
\hline SCS & Services capability server \\
\hline S-GWs & Serving gateways \\
\hline SGSN & Serving GPRS support node \\
\hline SMS-SC & Short message service-service center \\
\hline$\pi \mathrm{T}$ & Transmission time interval \\
\hline UMTS & Universal mobile telecommunications system \\
\hline WLAN & Wireless local area network \\
\hline WPAN & Wireless personal area network \\
\hline WSN & Wireless sensor network \\
\hline
\end{tabular}

smart metering/monitoring, smart lighting, smart grids, and telemedicine monitoring.

Several applications particularly related to intelligent and autonomous control are not yet considered as IoTbased applications [24]. However, developments in the domains of cloud computing and distributed multi-agent control along with WSNs and IoT have evolved M2M into a new form of communication called CPS. CPS has emerged as a prominent technology which is capable to support M2M and non-M2M applications. CPS offers communication and coordination among physical elements of a system and focuses on, e.g., interactive applications as well as distributed real-time control. CPS is an emerging form of IoT and can significantly benefit from communication technologies to provide services on the basis of information acquired from the surroundings (physical world). Figure 4 depicts the relationships and correlations among M2M, WSNs, IoT, and CPS [8]. The IoT is represented by the space formed by M2M, WSNs, and CPS. Additionally, Fig. 4 shows that developments in M2M and WSNs are expected to bring forth immense CPS applications in the future. Furthermore, authors concluded that currently M2M is considered as one of the crucial parts of IoT, whereas CPS is an evolution of M2M technology. Consequently, CPS will be regarded as one of the major technical frameworks for IoT.

\subsection{LTE/LTE-A architectural overview}

This section presents an overview of the LTE/LTE-A network architecture and protocols. Since this survey focuses on mobile M2M communication, an overview of the relevant LTE topics such as radio resource management, random access procedures, and UE power consumption is presented. Later on, these topics are thoroughly discussed in the context of mobile M2M communication challenges in Section 1.5.

\subsubsection{Long-Term Evolution}

Unlike traditional mobile networks such as global system for mobile communications (GSM), LTE is the first fully packet-switched mobile network proposed by 3GPP in 2004. 3GPP standardized LTE in its Release 8 with small modifications in Release $9[13,29]$. The standard is designed on the basis of the GSM and universal mobile telecommunications system (UMTS) networks to provide improved energy and spectral efficiency. The LTE radio and non-radio enhancements include evolved UMTS terrestrial radio network (E-UTRAN) and system architecture evolution (SAE), respectively. The evolved packet system (EPS) is the combination of LTE and SAE. The function of the EPS bearer ${ }^{2}$ is to route traffic from gateways (GWs) in the packet data networks (PDNs) to user equipments (UEs). A high level E-UTRAN architecture is presented in Fig. 5 (right). According to the 3GPP 


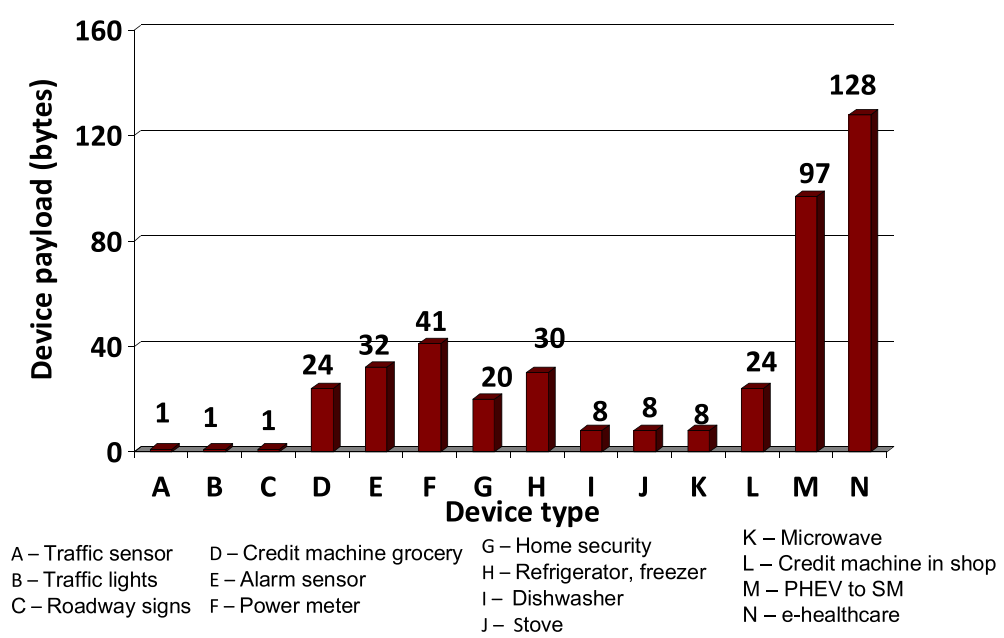

Fig. 3 Traffic models of various M2M applications representing small-sized data packets [142]

Technical Report TR 25.913 [30], the details of the performance requirements of 3GPP LTE networks can be described as follows:

1. LTE supports a maximum data rate of 100 and $50 \mathrm{Mbps}$ in downlink and uplink, respectively (for a 20-MHz bandwidth).

2. In downlink, the LTE mean throughput for a user is three to four times that of high-speed downlink packet access (HSDPA) (Release 6) per megahertz. In uplink, the average user throughput is two to three times that of high-speed uplink packet access (HSUPA) (Release 6) per megahertz.
3. The LTE control plane capacity supports approximately 200 active UEs per cell for a $5-\mathrm{MHz}$ bandwidth assignment.

4. The spectrum efficiency of LTE networks is three to four times that of HSDPA in downlink, whereas in uplink, it is two to three times that of HSUPA (Release 6).

5. Mobility support in LTE is $0-15 \mathrm{~km} / \mathrm{h}$ (low-speed users) and high-speed mobility support from $15 \mathrm{~km} / \mathrm{h}$ up to $500 \mathrm{~km} / \mathrm{h}$.

6. LTE supports a cell coverage of up to $5 \mathrm{~km}$ in order to meet throughput, spectral efficiency, and mobility

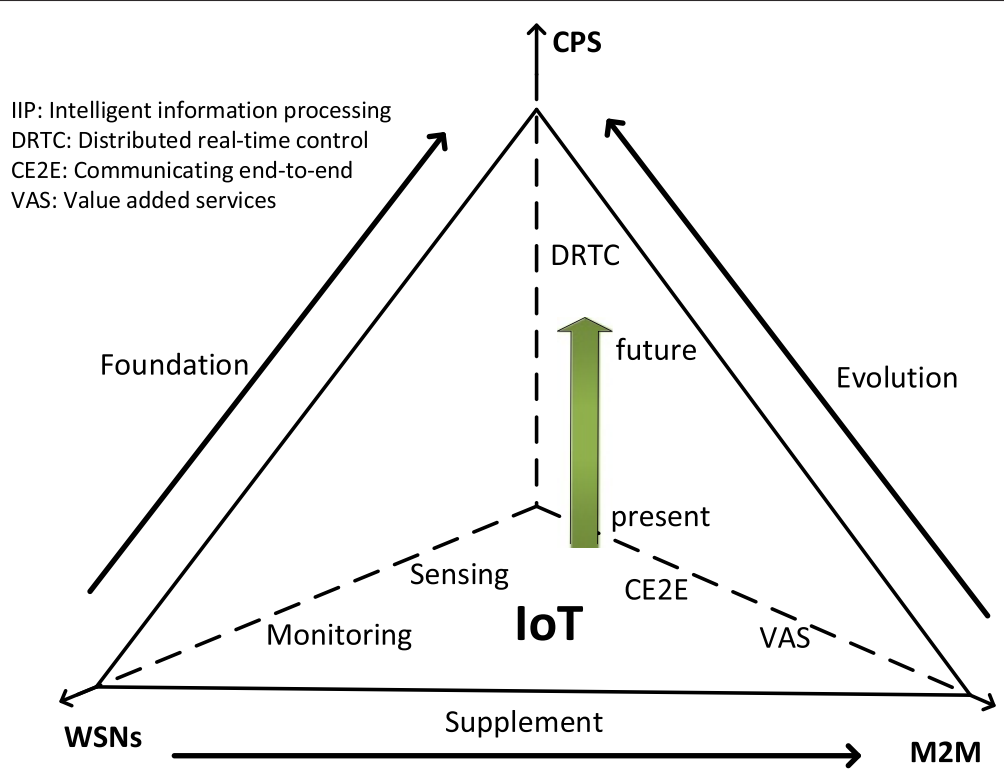

Fig. 4 A representation of correlations among M2M, WSNs, IOT, and CPS. Moreover, it shows that advancements in M2M and WSNs are expected to increase the number of CPS applications in the future (re-drawn from [15]) 


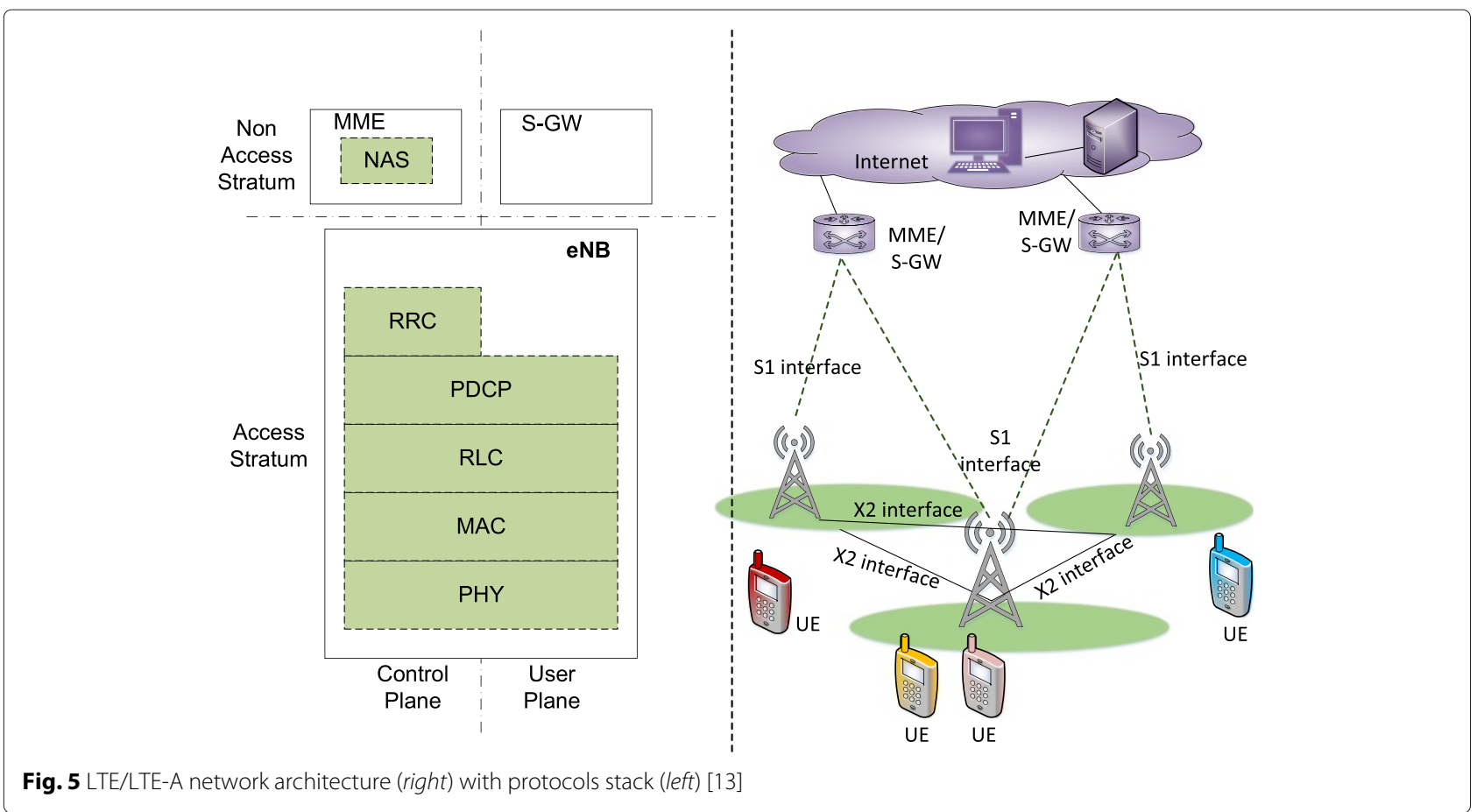

requirements. The cell coverage can be extended up to $30 \mathrm{~km}$ at the cost of minor performance degradation.

\subsubsection{LTE protocol overview}

LTE interfaces between UE, E-UTRAN, EPC, and S-GWs are associated with protocol stacks which are used to exchange data and signaling messages [31]. Therefore, the LTE protocol stack can be divided into the following categories:

1. User plane protocols: These protocols support routing of users data between UEs and S-GWs.

2. Control plane protocols: These protocols are used for exchanging signaling messages between various devices within the network.

On the air interface $(\mathrm{Uu})$, the UE high-level functionalities are controlled by the MME [32, 33]. However, there is no direct communication path between UE and MME. The communication path between UE and MME is established using the evolved node base stations (E-UTRAN eNB) which enhance the level of the hardware complexity in the network. To reduce this complexity, the Uu interface is further divided into two levels of protocols. One is called access stratum (AS) whereas the second is the nonaccess stratum (NAS). The MME high-level signaling lies in the NAS level but is transported within the network using AS protocols. The control and user plane termination protocols are supported by the eNB. The user plane protocols include the packet data convergence protocol (PDCP), the medium access control (MAC), and the physical (PHY) layer protocols. In addition, the protocols of the control plane include the radio resource control (RRC) protocols. A high level overview of the protocol stack is depicted in Fig. 5 (left). The major functionalities of these protocols are given below:

1. Access stratum (AS) [34]: UEs are capable to access the capabilities and services of communication networks through the AS protocols. There are several functionalities of the radio AS protocols such as dynamic allocation of radio resources to UEs (UL/DL), controlling bearers, traffic management, radio admission control, and handover management.

2. NAS: The NAS functionalities include the establishment of radio connections between the network and the UE. Moreover, registration, authentication, local registration management, and mobility management are also included in the functionalities of the NAS protocols.

3. RRC [35]: The major RRC functions and services include broadcasting system information, establishing/releasing RRC connections, security management, mobility functions, paging, QoS management, and direct messaging between UE and NAS.

4. PDCP [36]: The PDCP layer provides several services such as transfer of user and control plane data, header compression, re-transmissions of SDUs lost 
during handover, ciphering, detection of duplicate data, and in-sequence delivery.

5. Radio link control (RLC) [37]: There are several functionalities of the RLC layer such as the segmentation of data packets according to the available size of the transport block. Moreover, the error correction through automatic repeat request (ARQ) and the re-segmenting RLC data PDUs are included in the functionalities of the RLC. In addition, RLC performs functions such as concatenation, segmentation as well as reassembly of RLC SDUs, packet delivery, and error detection in protocols.

6. MAC [38]: The MAC layer functionalities include mapping between logical and control channels, RLC SDUs multiplexing/demultiplexing, scheduling, error corrections using hybrid ARQ (HARQ), priority handling of local channels, segmentation and reassembly of upper layer PDUs, padding, and ciphering.

7. PHY [39]: The PHY layer provides data transport services to the upper layers such as synchronization of time and frequency, physical channel modulation/demodulation, encoding/decoding of transport channels, MIMO operations, and transmit diversity. The frequency and time variation in LTE are exploited through orthogonal frequency-division multiplexing (OFDM) and single carrier frequency division multiple access (SC-FDMA) techniques. The physical layer sub-carrier spacing in LTE is $15 \mathrm{kHz}$.

\subsubsection{LTE radio resource management}

LTE transmission schemes support orthogonal frequency division multiple access (OFDMA) and SC-FDMA in downlink and uplink, respectively. The radio frame structure in the LTE uplink and downlink is similar. In LTE evolved universal terrestrial radio access (E-UTRA), the channel bandwidth ranges from 1 to $20 \mathrm{MHz}$ [40]. The E-UTRA channel bandwidths include 1.4, 3, 5, 10, 15, and $20 \mathrm{MHz}$. Each LTE frame $(10 \mathrm{~ms})$ is divided into ten subframes of $1 \mathrm{~ms}$ each [34], see Fig. 6. Every single subframe consists of two equal time slots of $0.5 \mathrm{~ms}$. Each slot is further divided into six and seven OFDM symbols in the extended and the normal cyclic prefix $(\mathrm{CP})$, respectively. For uplink and downlink communications in LTE, the smallest radio resource which can be allocated to each UE is called PRB which has a dimension of $180 \mathrm{KHz}$ in the frequency domain and $1 \mathrm{~ms}$ in the time domain. A single PRB is further composed of 12 consecutive sub-carriers. In normal cyclic prefix, there are 84 resource elements per PRB whereas an extended cyclic prefix is composed of 72 resource elements per PRB.

LTE radio resource management (RRM) enables efficient utilization of the scarce radio resources among several UEs. RRM consists of a set of procedures to distribute available PRBs among multiple users while ensuring their QoS as well as seamless connectivity [41]. The scheduling of radio resources in LTE is performed by the MAC layer of the eNB. Downlink and uplink scheduling procedures are initiated in every transmission time interval (TTI) of $1 \mathrm{~ms}$ depending on the amount of data in the buffers of eNB and UE, respectively. Furthermore, the RRM initiates the scheduling process when sufficient PRBs are available in the network to meet the QoS demands of other users as well. The transport block size (TBS) represents the capacity to transmit data with respect to the RRM procedure. In addition, the capacity of TBS depends on the available modulation and coding scheme (MCS) as well as the number of allocated PRBs to a particular UE. Figure 7 represents the capacity of TBS (in bits) with respect to the MCS index and the number of allocated PRBs. It can be seen from Fig. 7 that TBS capacity increases for higher values of MCS and larger number of allocated PRBs. For instance, 328 bits can be sent per PRB with an MCS index of 16. However, the PRB capacity increases to 712 bits when the index of MCS is raised to 26 .

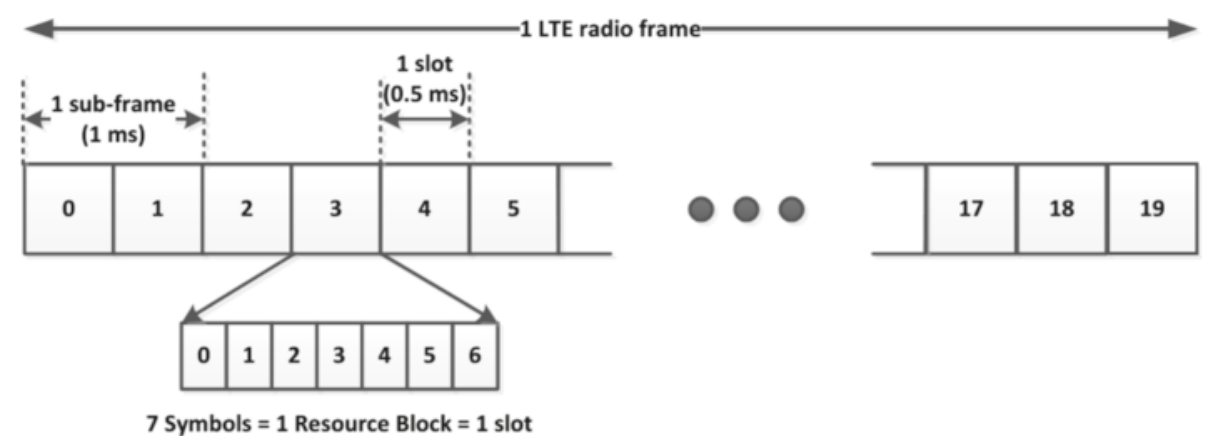

Fig. 6 LTE FDD frame structure [13] 


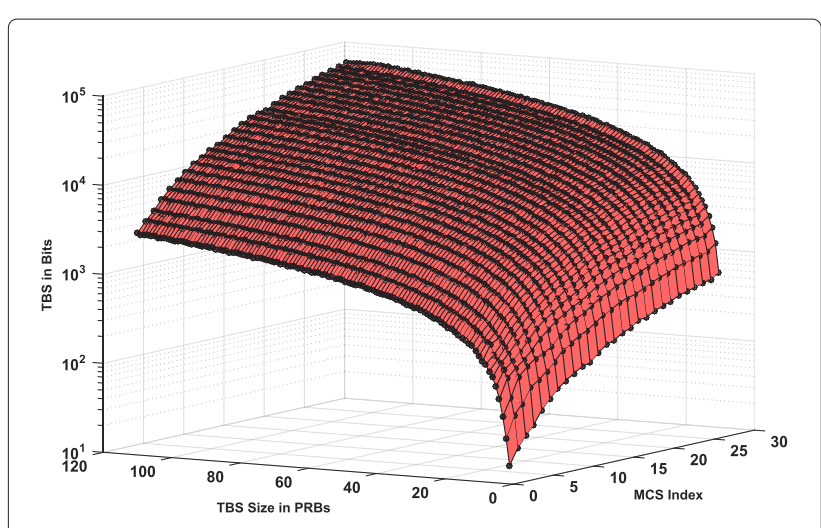

Fig. 7 An illustration of LTE TBS in bits with respect to MCS index and the number of allocated PRBs

\subsubsection{LTE random access mechanisms}

In LTE networks, each UE performs random access (RA) procedures to transmit data during the access grant time interval (AGTI) or RA slots over a time-frequency radio resource called physical random access channel (PRACH). A UE initiates the RA mechanism in two cases [42]. Firstly, when a UE is turned on and the uplink timing synchronization is either lost or not yet achieved. Secondly, whenever a handover mechanism is performed from a serving eNB to a target eNB. In LTE, UEs perform the RA procedure for several reasons such as for establishing a communication link, re-initiating a radio link after a failure, for radio resources in case of no uplink resource grant, and scheduling requests in the absence of dedicated physical uplink control channels (PUCCHs).
The RA procedure can be classified into a contentionbased and a contention-free random procedure [19, 4244]. Initially, a UE uses a contention-based RA procedure to select a preamble. A preamble is an OFDM-based signal with a narrower sub-carrier spacing. It is possible that several devices can select the same preamble, which demands a further contention resolution mechanism. In a contention-free RA procedure, an eNB uses dedicated preambles to initiate the RA mechanism. Hence, it ensures faster response than the contention-based approach. Contention-free RA procedures are usually used during critical situations such as handover. The major steps of contention-based and contention-free RA procedures are given in Fig. 8. Furthermore, a contention-free RA procedure is simpler than the contention-based RA procedure and consists of three steps by allocating dedicated preamble signatures to a device (see Fig. 8). The procedure starts with the RA preamble assignment by the eNB. After the transmission of an assigned RA preamble by the UE, the eNB responds with the random access response (RAR). This is the last step of the contention-free RA since there is no need to resolve further collision.

\subsubsection{UE power consumption in LTE}

Battery life optimization for mobile UEs, especially smartphone users, has always remained a challenging task for communication researchers. In the case of smartphones, users charge their phones frequently, usually every day. However, they always demand for the optimum power consumption of phones in order to increase its battery lifetime. In order to fulfill the increasing demand of improved UE power consumption, 3GPP introduced

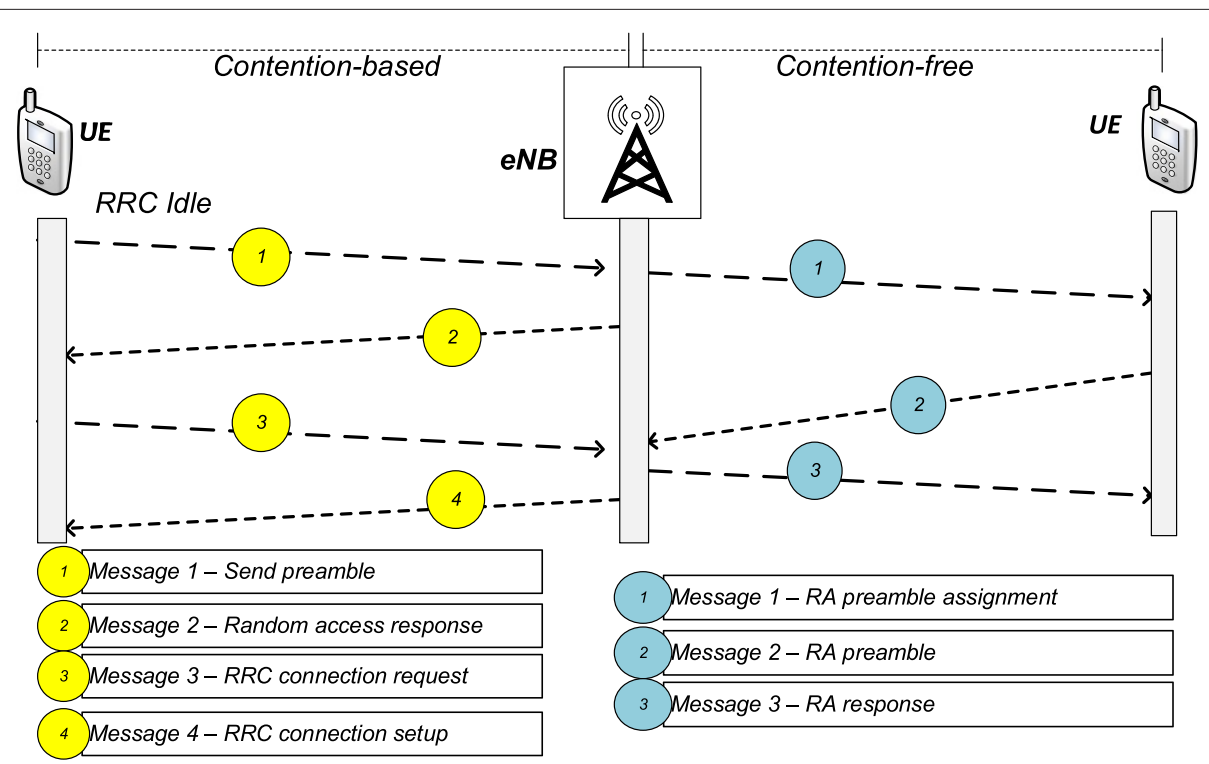

Fig. 8 An illustration of contention-based and contention-free RA procedures 
the power-saving mode (PSM) such as discontinuous reception (DRX) and idle state in its Release 12 TS 23.682 [45]. Under the PSM, a UE requests the network for an active timer duration during tracking area updates (TAUs) procedures. In this time, a UE remains attached, however all the AS layer functions are stopped. The DL data can be received by a UE in the RRC connected state, and during the active time period. The active timer is initiated by the UE when it moves from the connected to the idle state. The device moves to PSM with the expiry of the timer. The value of the active timer represents the time in which the device is accessible. In PSM, a device remains attached/registered with the network. However, it is not accessible due to the fact that it does not check for paging in PSM. Moreover, the device stays in PSM until it starts an activity such as UL data transmission. Figure 9 depicts the state transitions of a UE from connected to idle, and vice versa.

\subsubsection{LTE-Advanced}

LTE-A is the latest 3GPP standard which defines further advancements with respect to the existing LTE standard to approach the needs of IMT-Advanced which has been set by the International Telecommunication Union Radio communication (ITU-R) [46]. According to these requirements, LTE-A is capable to provide throughput of up to $1 \mathrm{Gbps}$ in downlink and $500 \mathrm{Mbps}$ in uplink. The peak spectrum efficiency in LTE-A is also improved by $15 \mathrm{Bps} / \mathrm{Hz}$ in uplink and $30 \mathrm{Bps} / \mathrm{Hz}$ in downlink with the maximum antenna configurations of $4 \times 4$ and $8 \times 8$, respectively. Moreover, the LTE-A standard is also designed to reduce the latency in user and control planes compared to the existing LTE standard. To achieve the defined tasks for LTE-A, the problems such as low signal-to-interference-plus-noise ratio (SINR) and network coverage issues due to shadowing and non-lineof-sight (NLOS) are considered. According to [47], the capacity of the 3GPP LTE Release 8 network is already very close to the Shannon limit and it is required to upgrade the existing technologies with new standards to support the increasing future data traffic. One idea is to increase the number of eNBs which is not a cost-efficient approach and not an appealing option for network operators.

In LTE-A networks, the evolved UMTS terrestrial radio network (E-UTRAN) consists of eNBs with a similar role as that of the base station (BS) in GSM but with additional functionalities [41, 48]. The E-UTRAN handles communication between the UE and the EPC, whereas an eNB handles a UE in one or more cells. The serving eNB (SeNB) is the eNB which currently provides services to a UE. Moreover, the eNB controls low-level operations such as handover functions. The eNB communicates with the core network through the S1 interface. Several eNBs are also inter-connected with each other through the $\mathrm{X} 2$ interface (see Fig. 5, right) which is mainly used for exchanging information such as packet forwarding during handover. The S1 interface between an eNB and EPC can also perform handover functions at the cost of more delay. The S1 and X2 interfaces operate using an IP-based transport network. Moreover, E-UTRAN also provides home eNB (HeNB) services. HeNB is an eNB which is

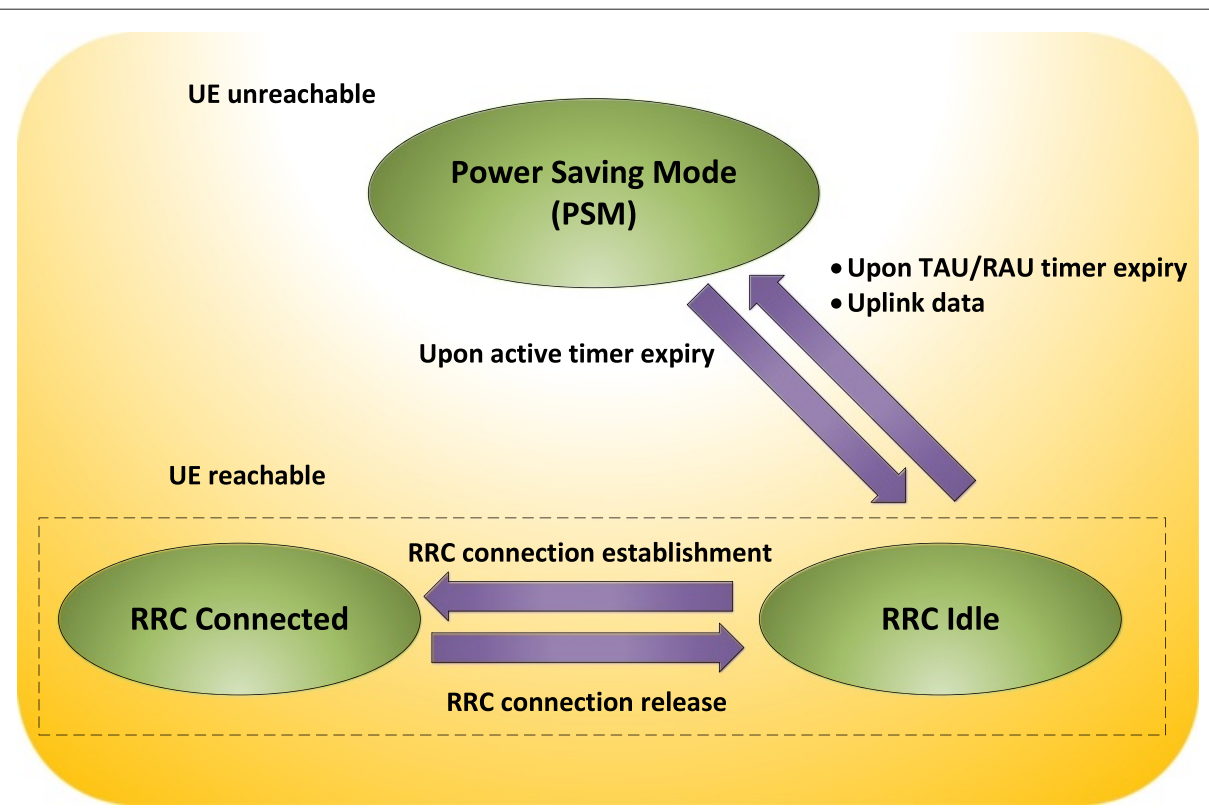

Fig. 9 A graphical representation of 3GPP standardized PSM 
particularly dedicated to a user within a home for femtocell coverage. It can be connected either directly to the $\mathrm{EPC}$ in the same way as a standard eNB is connected or using some intermediate terminals such as gateways that forward information from multiple eNBs.

\section{$1.4 \mathrm{M} 2 \mathrm{M}$ network architectures}

Besides the already mentioned characteristics of M2M traffic, it also exhibits unique characteristics such as an increasing M2M device volume, sending small payloads, the demand for various mobility profiles, time-controlled, and mainly delay-tolerant as well as bursty traffic [26]. Therefore, it is of significant importance to optimize the existing mobile standards which have been originally standardized for traditional mobile traffic. Due to the abovementioned unique characteristics, M2M communications demand an optimized E2E network architecture, particularly over mobile networks such as LTE/LTE-A. To enable global connectivity among various M2M applications, several standardization bodies are working on designing new and optimized architectures to support M2M communications over mobile networks. ETSI has designed and standardized an E2E network architecture for M2M communications by considering various M2M service requirements. Similarly, 3GPP has also defined a reference model for MTC. In this section, both ETSI and 3GPP M2M architecture and reference models are discussed for the understanding of the reader.

\subsubsection{ETSI M2M architecture}

According to ETSI specifications discussed in [15, 49, 50], the M2M architecture consists of the following five key elements which are shown in Fig. 10:

M2M device domain The M2M devices are responsible for the collection and autonomous transmission of sensor data such as the internal temperature and humidity level of a container in logistics, and ITS. The M2M devices are usually connected to small local networks called subnets for the transmission or reception of data to or from the $\mathrm{M} 2 \mathrm{M}$ application domains (server domain).

M2M area networks The M2M area networks are responsible for establishing the communication path between the M2M devices and the M2M gateways. These

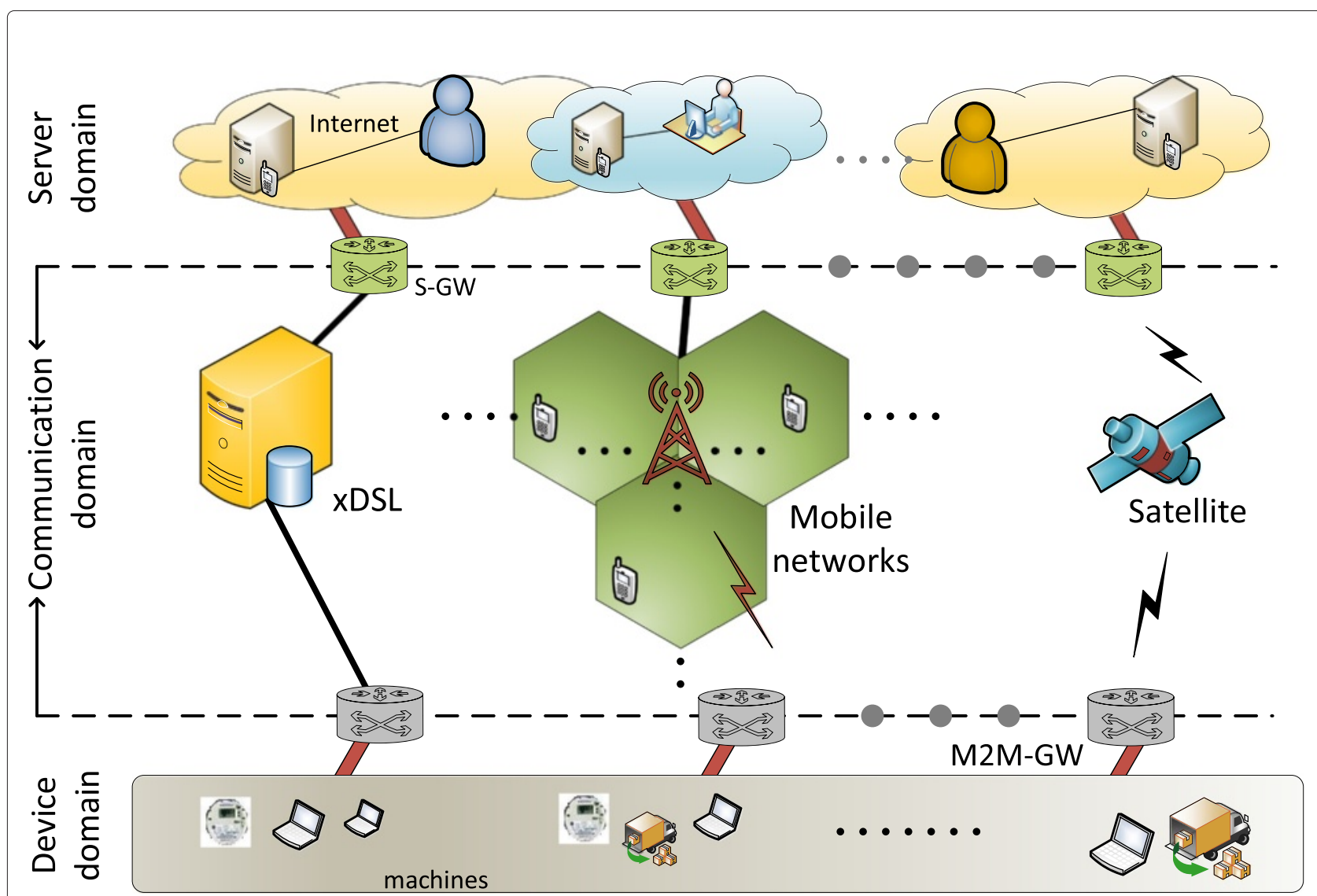

Fig. 10 A high-level E2E network architecture for M2M communications proposed by ETSI (re-drawn from [15]) 
networks are usually called subnets which collect and route information from the M2M devices to the M2M gateways. There are several subnets which are used for generating the communication link between the M2M devices and the M2M gateways [50]. Generally, the use of subnets is dependent on the network technology. In fully distributed networks, all M2M devices are connected as peers to the network. One of the nodes which is connected to the network, e.g., via wired or wireless connectivity, acts as a router. In client-server networks, all nodes or devices communicate directly with the server. Whereas in cooperative networks, all nodes communicate with each other using some intermediate gateways. Some of the major subnets are presented in Table 2.

M2M gateways The intelligent sensors, which are deployed to collect information, communicate with the communication network with the help of M2M gateways. Hence, M2M gateways act like bridges between the sensors and the communication network.

Communication networks The main role of the communication network is to create a communication path between the M2M devices and application servers either through wired or wireless communication networks. The main communication networks are listed in Table 3.

Server/application domain The M2M application domain consists of a middleware layer where the collected packets pass through several application services and then are used by the related agencies.

\subsubsection{GPP MTC reference model}

In the 3GPP MTC reference model given in [45, 51-53], the communication between MTC devices and application servers use 3GPP services and optionally services capability server (SCS $)^{3}$ services. The application server (AS) particularly hosts an MTC application in the

Table 2 List of technologies for M2M communication [15]

\begin{tabular}{ll}
\hline Sr & M2M area networks \\
\hline 1 & Power line communication (PLC) \\
2 & Short-range device (SRD) \\
3 & Ultra wide band (UWB) \\
4 & ZigBee \\
5 & Meter bus (M-BUS) \\
6 & Wireless meter bus \\
7 & Bluetooth \\
\hline
\end{tabular}

external network. Furthermore, the AS manipulates an SCS for supplementary value-added services. The 3GPP provides several services to support MTC, e.g., transport, user management as well as architectural enhancements, e.g., initializing control plane functionalities of an MTC device [52]. 3GPP envisages various models for MTC communications [52]. These models are related to the SCS provider-based communication between the AS and the 3GPP system. The various architectural models proposed by 3GPP include the direct model, the indirect model, and the hybrid model $[45,52,53]$. In the case of the direct model, the AS directly performs data communication with the UE by connecting directly to the network and does not use any SCS service (see Fig. 11). In addition, the 3GPP services can be used by the application in the outer network. In the case of the indirect model, the AS uses SCS services and connects indirectly to the network. Thus, the indirect model utilizes supplementary value-added services for MTC such as control plane device triggering. In addition, the SCS is either controlled by the MTC service providers or network operators in order to provide several services, e.g., value-added services and communication services related to control and user plane. In case of the hybrid model, both the aforementioned models (direct and indirect) are used by the AS concurrently. The AS performs direct data communications with the device by connecting directly to the network. However, it also uses SCS services. From the 3GPP network aspect, the AS direct data communication and the SCS value-added services, e.g., control plane communication are fully independent, although both can service the same AS MTC application. In addition, the MTC applications can receive distinct services/facilities from the SCS controlled by the MTC service provider as well as the 3GPP operator. Since the abovementioned three models are complementary, 3GPP operators can combine them for various applications. For instance, the SCS based on the MTC service provider can be combined with the 3GPP network operator controlled SCS, both communicating with the similar PLMN.

Table 3 List of technologies for M2M communications [15]

\begin{tabular}{ll}
\hline $\mathrm{Sr}$ & M2M communications networks \\
\hline 1 & Short-range devices (SRDs) \\
2 & Ultra wide band (UWB) \\
3 & Satellite \\
4 & GSM, UMTS, LTE, LTE-A \\
5 & Wireless LAN \\
6 & WiMAX \\
7 & XDSL \\
\hline
\end{tabular}




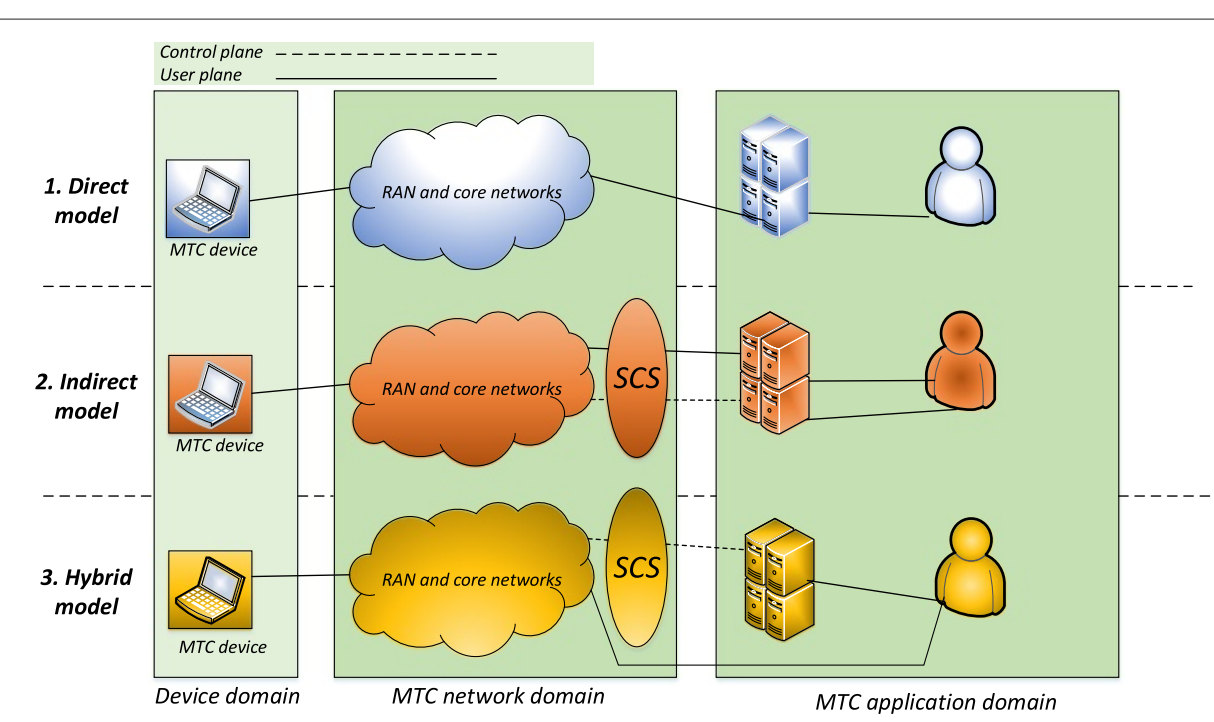

Fig. 11 GGP direct, indirect, and hybrid models for MTC communications

Architectural reference model The architectural reference model for MTC is depicted in Fig. 12, which shows an MTC device communicating with 3GPP networks, i.e., E-UTRA, E-UTRAN, and GERAN using Um, Uu, and LTE-Uu interfaces, respectively. Moreover, Tsp is used for 3GPP network services to SCS and AS (see Fig. 12). 3GPP has standardized the Tsp interface in order to support value-added services for MTC such as control plane device triggering. In addition, an SCS usually provides Tsp interface. Moreover, the MTC interworking function (MTC-IWF) is a functional entity used to support indirect and hybrid models by residing in the home public land mobile network (HPLMN). It usually shows the internal PLMN network topology. Furthermore, it also translates Tsp-related signaling protocols to bring particular functionality in the PLMN. The descriptions of various MTC-related reference points and network elements are summarized in Table 4 (taken from [53]).

\subsubsection{MTC service requirements in mobile networks}

The service requirements vary significantly due to the diversity of MTC applications. For this reason, the authors of [15] and [54] stated that MTC traffic types significantly depend on the type of technologies used for communication between devices and MTC application domains. This subsection presents MTC service requirements by focusing on 3GPP networks.

3GPP MTC features The MTC traffic characteristics differ significantly from application to application. Resultantly, system as well as service optimizations also vary, thus a particular optimization approach might not be applicable to the vast M2M application range [55]. 3GPP has defined a set of features for MTC in order to provide a structure to optimize systems according to varying MTC applications. In general, 3GPP identifies the following MTC features in its technical specifications TS 22.368 [55]:

1. Small-sized data transmissions

2. Infrequent mobile and data termination

3. Device low-to-high mobility

4. Time-controlled traffic behavior

5. Group-based policing and addressing

6. Ensuring secure connections and transmissions

7. MTC device monitoring requirements

3GPP MTC common service requirements $3 \mathrm{GPP}$ has set certain service requirements for MTC in its Release 10 and onward. The major 3GPP-based MTC service requirements are summarized below (taken from [55-58]):

1. The system shall support a large number of MTC devices, i.e., connectivity for massive number of MTC devices.

2. The system shall provide an efficient resource allocation scheme to support MTC devices which send or receive small sized data, i.e., with longer inter-arrival/inter-send time.

3. The system shall provide an option for an MTC user for activating or deactivating MTC features.

4. The networks shall enable service providers to identify which MTC features are activated for a particular MTC user. 


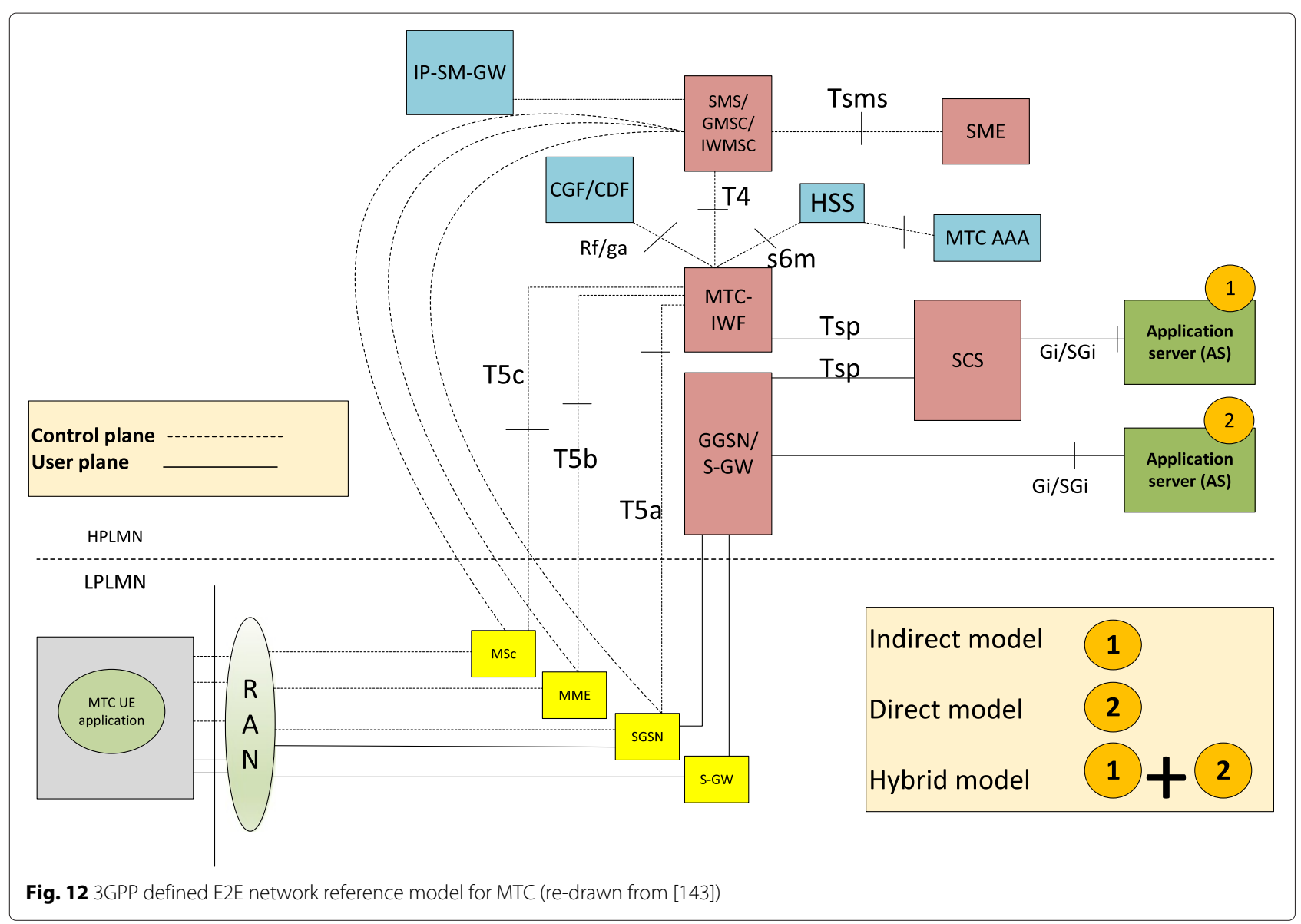

5. In the case of overload, the network shall restrict downlink transmissions and signaling.

6. In the case when a large number of devices attempt to access the network, the system shall support a scheme to limit peaks in the data as well as in signaling traffic.

7. If the network is overloaded, the system shall be capable to limit access towards a particular access point name (APN).

8. Each MTC device shall be capable to support the extended access barring (EAB) scheme defined in 3GPP TS 22.011 [27].

9. The system shall support methods to ensure low power consumption of an MTC device in order to increase its lifetime.

10. If MTC devices are not sending or receiving data, then the devices can or cannot be kept attached to the networks, depending on operator policies and requirements of an MTC application.

MTC device triggering 3GPP-based device triggering requirements of an MTC device are summarized as follows $[55,58]$ :
- Whenever an MTC server shows a trigger, an MTC device shall initiate communication with an MTC server.

- The network shall ensure that an MTC server's triggering indications to trigger MTC devices is authentic.

- In case of triggering from a source which is not authentic, the network shall be able to report to the MTC device by providing the details of fictitious/malicious source.

- The network should provide a mechanism to give information to MTC devices regarding authentic servers or sources.

- The MTC device shall create a link to communicate with an MTC server. Therefore, an MTC device shall receive a trigger whenever it is indicated by an MTC server. There can be three possibilities, i.e., receiving trigger indications while an MTC device is not attached to the network, receiving trigger indications when an MTC device is attached to the network without transmitting/receiving data, and receiving trigger indications when the MTC device is 
Table 4 List of the reference points and network elements of the 3GPP reference model for MTC communications (taken from $[45,51-53])$. The acronyms of network elements are given in Table 1

\begin{tabular}{|c|c|}
\hline & Descriptions \\
\hline \multicolumn{2}{|l|}{ Reference points } \\
\hline \multirow[t]{2}{*}{ Tsms } & It enables the communication between an entity lying outside the 3GPP network and \\
\hline & MTC using SMS \\
\hline \multirow[t]{2}{*}{ Tsp } & It connects MTC-IWF to SCSs to provide several functionalities \\
\hline & such as device triggering \\
\hline \multirow[t]{2}{*}{ T4 } & It performs SME tasks, e.g., connects MTC-IWF to the HPLMN domain. \\
\hline & T4 also supports device triggering functionlities \\
\hline $\mathrm{T} 5 \mathrm{a}$ & It enables communication between MTC-IWF and serving SGSN \\
\hline T5b & It establishes the communication path between MTC-IWF and serving MME \\
\hline $\mathrm{T} 5 \mathrm{c}$ & It creates the communication link between MTC-IWF and serving MSC \\
\hline \multirow[t]{2}{*}{ S6m } & It performs subscription and MTC-related information tasks by establishing \\
\hline & the communication path between MTC-IWF to HSS/HLR \\
\hline \multirow[t]{3}{*}{ S6n } & It also performs subscription and MTC-related information tasks by establishing \\
\hline & communication link between MTC-AAA and HSS/HLR and supports \\
\hline & interrogation of HSS/HLR \\
\hline \multicolumn{2}{|l|}{ Network elements } \\
\hline \multirow[t]{3}{*}{ MTC-IWF } & One of the instances of MTC-IWF exists in HPLMN to support indirect and hybrid models \\
\hline & Receives trigger request of an MTC device from SCS \\
\hline & Reporting and updating SCS about the acceptance or rejection of the device trigger request \\
\hline \multirow[t]{2}{*}{$\mathrm{HSS} / \mathrm{HLR}$} & It determines whether an SCS is allowed to send a trigger to a particular MTC \\
\hline & It also performs mapping tasks between IMSI and external identifier(s) \\
\hline GGSN/P-GW & It enquires an MTC AAA server for regenerating external identifier(s) \\
\hline \multirow[t]{5}{*}{ SGSN/MME/MSC } & Accepting an MTC device trigger from MTC-IWF \\
\hline & It confines the device trigger information in NAS messages sent to MTC \\
\hline & It accepts a device trigger acknowledgement from the triggering MTC \\
\hline & It reports to the MTC-IWF about the device trigger delivery success/failure status \\
\hline & It also delivers SGSN/MME congestion/load information to MTC-IWF \\
\hline SMS-SC & It delivers the routing information from MTC-IWF to SMS-GMSC \\
\hline MTC AAA & It uses standard procedures such as RADIUS to identify the destination external AAA server \\
\hline \multirow[t]{2}{*}{ SCEF } & It performs several tasks such as device authentication and profile management \\
\hline & It makes external entities capable to discover various service capabilities \\
\hline
\end{tabular}

attached to the network with an established data connection.

MTC addressing and identifiers [55, 58] Each MTC device shall be assigned a private IPv4 address by the mobile network operator (MNO) if the MTC server exists in a public IPv4 address space. Regarding identifiers, the system shall uniquely recognize the attached MTC users. Furthermore, the system shall present techniques for the network operator to efficiently manage MTC-related numbers as well as identifiers.

\subsubsection{Standardization efforts for M2M communications}

According to [15], M2M communications significantly rely on numerous technologies spanning across several industries. Due to the wide application range, standardization in M2M communication is more challenging than the traditional standardization for a particular platform. Consequently, the required standardization scope is much higher than the existing standardization developments and entails to upgrade/modify existing standards to support future M2M traffic on a large scale. 3GPP, ETSI, IEEE, and Telecommunications Industry Association (TIA) have played a significant role in developing 
new standards to support M2M communications. The standardization work related to $\mathrm{M} 2 \mathrm{M}$ communication is on-going in several standardization organizations outside 3GPP (e.g., ETSI, IEEE, TIA, and oneM2M). Moreover, these standardization bodies have also described the required enhancements in the existing communication standards in their specifications. One important effort for M2M standardization in 2012 is the oneM2M partnership by the global standard development organizations (SDOs) [59].

This section presents the major standardization efforts made by several SDOs to facilitate M2M communications and services. The major efforts made by several standardization bodies are described below. Moreover, a summary of their major contributions is given in Table 5.
oneM2M [60] oneM2M is a global initiative to support future M2M communications. Eight of the world's primary ICT standardization organizations such as ETSI and TIA have initiated oneM2M. Under this oneM2M umbrella, six of the globally active industry consortia such as broadband forum, OMA, and Continua have joined the regional standards bodies for ensuring efficient M2M communications. Other than the standards bodies and industry consortia, around 200 members from universities, research labs, and big companies have also joined this initiative. One of the primary objectives of oneM2M is to develop a technical design which should fulfill the requirements for a general service layer platform for M2M communications. Consequently, the designed specifications can be easily implanted within several hardware- and software-based systems to offer connectivity for a massive

Table 5 Summary of contributions made by the major standards development organizations to support M2M communications

\begin{tabular}{|c|c|c|}
\hline SDOs & Contributions & Reference \\
\hline \multirow[t]{3}{*}{ oneM2M } & - M2M functional architecture, requirements, and security solutions & {$[146,147]$} \\
\hline & - Service layer core protocol specification & [148] \\
\hline & - Protocol binding (COAP, HTPP, and MQTT) & [149-151] \\
\hline \multirow[t]{5}{*}{ 3GPP } & - MTC reference model & [51] \\
\hline & - MTC service requirements & [51] \\
\hline & - Radio resource management for MTC & {$[10,61]$} \\
\hline & - Considering MTC security aspects & {$[62,63]$} \\
\hline & - Describing MTC-use cases & {$[61]$} \\
\hline \multirow[t]{7}{*}{ ETSI } & - M2M networks architecture and technologies & {$[28,49,65]$} \\
\hline & - QoS for M2M communications & {$[67]$} \\
\hline & - M2M service requirements & {$[67]$} \\
\hline & - M2M-use cases: e-healthcare, smart metering, automotive, and smart grid & [69-72] \\
\hline & - Several M2M-related interfaces, i.e., mla,dla, and m/d & {$[66]$} \\
\hline & - M2M data semantic support & [152] \\
\hline & - SmartM2M test specifications: interoperability for ETSI M2M primitives & {$[68]$} \\
\hline \multirow[t]{3}{*}{ IEEE } & - Optimizing air interface & {$[73,74]$} \\
\hline & - Use of sub-GHz spectrum for M2M communications & {$[74]$} \\
\hline & - Optimizing air interfaces for the smart grid IEEE 802.15.4 & {$[74]$} \\
\hline \multirow[t]{3}{*}{ GSMA } & - $\mathrm{M} 2 \mathrm{M}$ resource management schemes & {$[77]$} \\
\hline & - M2M-related protocols and interfaces & {$[77]$} \\
\hline & - M2M communications using remote SIM & {$[77]$} \\
\hline CCSA & - Supporting smart grid applications & {$[81]$} \\
\hline \multirow[t]{2}{*}{ WFA } & - Integrating Wi-Fi in smart grids & {$[79]$} \\
\hline & - e-healthcare application scenarios & [79] \\
\hline \multirow[t]{2}{*}{ WiMAX } & - M2M deployment scenarios & {$[78]$} \\
\hline & - Functional and network improvements & {$[78]$} \\
\hline \multirow[t]{2}{*}{ OMA } & - M2M device management according to ETSI remote entity management & {$[80]$} \\
\hline & - Personal area networks for ensuring efficient capillary M2M communications & {$[80]$} \\
\hline
\end{tabular}


number of M2M devices to the backend server worldwide. Additionally, oneM2M focuses on to influence other organizations offering numerous M2M services in the perspective of business such as ITS, logistics, e-healthcare/mhealthcare, industrial automation, and smart metering and monitoring.

3GPP 3GPP has made progress for network developments for inter-MTC device communication in Release 12. 3GPP proposed several solutions to handle large numbers of MTC devices in GSM and UMTS networks. The study focused on handling a massive number of MTC devices which are expected to communicate either directly with each other or with the backend server simultaneously. Moreover, the service requirements for MTC are addressed in [54]. Several key issues such as network congestion due to the large number of devices are discussed. In addition, numerous possible solutions such as group-based communication are given in order to tackle the aforementioned issues. 3GPP in its Release 12 [61] described various enhancements as well as use cases for MTC. Several applications for MTC such as transportation and logistics, surveillance, and e-healthcare are addressed. The security aspects in MTC are addressed in [62] and [63]. Furthermore, a detailed study of RAN enhancements and GERAN improvements for MTC is also given.

In addition to the aforementioned contributions, 3GPP started interacting with third parties in [64] and empowered them to facilitate users through third party services using 3GPP functions. In order to accomplish it, third parties also enable MTC services after making an agreement with operators. Furthermore, 3GPP has started working on an MTC architecture in Release 10. In Release 11, 3GPP defined several interfaces between the core network $(\mathrm{CN})$, MTC service, and application enablement platforms. 3GPP is currently working on small data transmissions and low power consumption devices in Release 12 SA2. The major existing and expected efforts to support MTC in upcoming Releases are summarized in Table 6.

ETSI ETSI has made serious efforts in developing standards and protocols for M2M communication. ETSI has established a new committee with goals to provide an overall M2M communication overview. Moreover, the group has also focused on relating M2M communication closely with the ETSI development for the latest communication standards. Moreover, ETSI focuses on to relate $\mathrm{M} 2 \mathrm{M}$ communication with the advanced 3GPP new communication technologies. The detailed E2E network architecture for M2M communication by ETSI is given in [28]. Furthermore, a detailed study of the intercommunication of the proposed ETSI M2M architecture
Table 6 Overview of 3GPP MTC standardization efforts (based on [145])

\begin{tabular}{ll}
\hline Parameters & Work items \\
\hline 3GPP Release 11 (2012) & - Power preference indications \\
- Random access control overload & - Cat-0 MTC devices (low cost) \\
- PGPP Release 12 (2014) & - Device assisting information for \\
& eNB tuning \\
3GPP Release 13 (expected in 2016) & - Low-cost MTC modules \\
& - Coverage enhancements \\
& - Power-saving enhancements for \\
& eNB tuning
\end{tabular}

with local area networks such as ZigBee is given in [65]. In addition, several communication interfaces such as mla, $d l a$, and $m l d$ for M2M communications are provided in [66]. Moreover, the future requirements for M2M communication such as mobility support are discussed in [67]. Test specifications for the constraint application protocol (CoAP) binding of M2M primitives is presented in [68]. In addition, several use cases of M2M communication such as e-healthcare and smart metering are explained in [69] and [70], respectively. Moreover, M2M automotive applications and smart grid applications are discussed in [71] and [72], respectively.

IEEE IEEE has started studying M2M communication by announcing working groups for M2M communication $[73,74]$. The working group IEEE 802.16p 10/0005 describes the usage of M2M communication and various standard modifications which are required to support future M2M communications. In addition, the working group IEEE $802.16 p-10 / 0014$ provides various methodologies which seem to be helpful for M2M communications. Furthermore, IEEE also focuses on air interface optimization for mass device communication, the use of sub-GHz spectrum (Wi-Fi) for M2M communication, and optimizing air interfaces for the smart grid IEEE 802.15.4.

TIA [75] This SDO provides bi-directional communication between smart devices and the networks in its TR-50 framework [76]. Furthermore, it provides an extension for e-healthcare scenarios in which e-healthcare sensors can access the converged personal network service (CPNS) server in the global network via a mobile gateway. This extension in e-healthcare provides monitoring and simple daily care services. Hospitals and pharmacies can access information received from the sensors deployed on the body of the patient. 
GSM Association (GSMA) [77] This SDO focuses on GSM-based M2M communication in which the major developments include module design, radio interface, and authentications.

Worldwide Interoperability for Microwave Access (WiMAX) [78] This SDO covers developments mainly in deployment models, functional improvements, and network improvements.

Wi-Fi Alliance (WFA) [79] WFA covers integration of Wi-Fi within smart grids and e-healthcare cases.

Open Mobile Alliance (OMA) [80] The key enablers for M2M devices and services include M2M device management, M2M gateway management, personal area network services to fulfill ETSI M2M area network requirements, and location detection services [80].

\section{China Communications Standards Association} (CCSA)[81] CCSA focuses on standards for interfacing smart grids and location-based services.

\subsubsection{Major research projects}

Due to the emerging use of mobile networks for M2M communications, several research projects such as EXpAnding LTE for Devices (EXALTED) and Achieving LOw-LAtency in Wireless Communications (LOLA) focused M2M communications for supporting increasing M2M traffic efficiently. Furthermore, several of the latest EU research projects such as METIS-2020 have extensively addressed existing and upcoming M2M-related issues in their research. Consequently, several recommendations and solutions have been proposed in order to handle M2M small-sized transmissions efficiently on a large scale. METIS-2020, one of the largest EU research projects addressed $\mathrm{M} 2 \mathrm{M}$ upcoming issues as a front line research topic, within the list of its horizontal topics. Resultantly, several solutions have been proposed for handling future mobile M2M traffic efficiently. This subsection presents an overview of these projects and highlights their major contributions, for the ease of the reader to understand the existing and upcoming issues and research gaps. In the end, a summary of all major contributions related to M2M communications is given in Table 7.

METIS-2020 [82] Mobile and Wireless Communications Enablers for the Twenty-Twenty Information Society (METIS-2020) is an EU research project, initiated in 2013. Twenty six partners including major operators such as Ericsson, NTT Docomo, and NOKIA have participated in this project. The primary goal of this project is to lay the foundation of upcoming $5 \mathrm{G}$ mobile and wireless systems.
Since cellular-based M2M communications is one of the latest research topics, several massive machine communication (MMC)-related issues are addressed in METIS [83]. Furthermore, future MMC scenarios are discussed, and consequently several novel radio link schemes are proposed to handle the increasing mobile M2M traffic. Since handling $\mathrm{MMC}$ in future mobile networks is a challenging task especially for the radio link layer, METIS focused on providing multiple access and multiple control schemes, advanced MAC and HARQ algorithms, synchronization issues, and MMC-related signaling limitations for MMC scenarios in its radio link analysis.

EXALTED [3] EXALTED is an EU FP7 ${ }^{4}$ ICT research project commenced in 2010 (September) for a duration of 30 months. The project was coordinated by Sagemcom SAS, France, along with the other 12 partners including well-known operators and module manufacturers such as Vodafone, Ericsson, and Gemalto. One of the key issues of EXALTED is to provide secure, cost- and energyefficient M2M communications in the context of future mobile technologies. Therefore, EXALTED also considered existing challenges in mobile networks to finally deliver the foundation of a new scalable network architecture. Furthermore, the project aimed at the specifications of massive machine communications under the name of $L T E-M$ networks particularly in mesh networks. The resulting system provides a low cost and spectrally efficient M2M system with extended coverage in LTE networks. Approximately 1000 devices were expected to be supported per cell with the proposed architecture and schemes by assuming quite relaxed latency requirements greater than $100 \mathrm{~ms}$.

LOLA [84] LOLA is another major EU FP7 research project coordinated by EURECOM, France. The project focused on access layer enhancements to support M2M communications in mesh networks with medium coverage as well as in mobile networks such as LTE-A with long range. LOLA aimed to ensure efficient spectrum utilization by targeting a particular set of applications with low latency requirements such as gaming. In cellular networks, the project focused on improving transmission technologies for supporting gaming applications which is expected to be one of the crucial areas for operators to increase their revenue in the future. Therefore, the M2Mrelated research focused to deliver recommendations to 3GPP regarding PHY/MAC functionalities in support of M2M. LOLA provided inputs to 3GPP standardizations directly as well as targeting the improvement of LTE MAC and PHY layers by designing schedulers, framing, adaptive modulation/coding schemes, as well as ACK/NACK feedback. 
Table 7 Summarizing contributions of the major research projects for M2M communications

\begin{tabular}{|c|c|c|c|}
\hline Project name & Duration & Contributions & Reference \\
\hline \multirow[t]{7}{*}{ METIS 2020} & \multirow[t]{7}{*}{ Nov. 2013-May 2015} & - Scenarios, requirements, and KPIs for future M2M & {$[86]$} \\
\hline & & - Requirements for new air interfaces & [83] \\
\hline & & - Novel radio link concepts for M2M & [153] \\
\hline & & - Radio resource allocation techniques & [153] \\
\hline & & - M2M traffic offloading ideas/low signaling overhead & [154] \\
\hline & & - M2M via D2D communications & [153] \\
\hline & & - M2M massive random access & [154] \\
\hline \multirow[t]{8}{*}{ EXALTED } & \multirow[t]{8}{*}{ Sept. 2010-Mar. 2013} & - Impact of M2M applications on business models & [155] \\
\hline & & - LTE-M algorithms and procedures & {$[156,157]$} \\
\hline & & - EXALTED M2M system architecture & [158] \\
\hline & & - Optimizing a Linux operating system for M2M & [159] \\
\hline & & - Device management & [160] \\
\hline & & - Capillary M2M traffic aggregation & [161] \\
\hline & & - Security solutions for M2M & [162] \\
\hline & & - Security solutions for P2P relaying & [163] \\
\hline \multirow[t]{8}{*}{ LOLA } & \multirow[t]{8}{*}{ Oct. 2010-Mar. 2013} & - Evaluation of M2M application scenarios & [164] \\
\hline & & - Access layer techniques for low latency robust transmissions & [165] \\
\hline & & - Spectral efficiency with M2M & [166] \\
\hline & & - M2M in long-range and short-range mesh networks & [165] \\
\hline & & - Transmission technologies for M2M gaming services & {$[165,167]$} \\
\hline & & - $\mathrm{PHY} / \mathrm{MAC}$ design procedures, algorithms, and scheduling & {$[165,167]$} \\
\hline & & - Characterization and modeling of traffic & [167] \\
\hline & & - Experimentation traffic measurement testbench etc. & [168] \\
\hline
\end{tabular}

\subsection{Mobile MTC challenges and solutions}

Existing mobile networks such as LTE were originally designed and subsequently upgraded due to the tremendous growth in mobile data traffic. On the one hand, the CISCO 2014-2019 forecast [85] shows that mobile data traffic will grow to 24.3 exabytes (EB) per month by 2019 , up from $2.5 \mathrm{~EB}$ in 2014 . On the other hand, $\mathrm{M} 2 \mathrm{M}$ traffic is expected to increase even more rapidly as compared to traditional mobile traffic, making QoS provisioning a huge challenge. Consequently, current mobile networks will not be able to support the increasing number of M2M devices worldwide [83]. M2M traffic poses serious challenges to cellular operators due to the high density of devices transmitting small-sized payloads [86]. For instance, according to the latest M2M traffic forecasts, GSMA 2020 forecasts that the number of cellular-based M2M devices will grow to one billion by 2020 [87]. Furthermore, approximately 3.2 billion cellular-based M2M devices are expected in 2024 (M2M Magazine 2014-2024 forecast [88]). This rapidly increasing M2M devices would undoubtedly cause severe challenges for mobile networks. The major challenges for mobile networks include the following:
- Support for a large M2M device volume

- Small-sized data transmission after regular as well as irregular time intervals

- Ensuring low power consumption as compared to non-cellular standards

- Low latency, high reliability, and support for various mobility profiles

- Ensuring that M2M traffic is not affecting regular $\mathrm{H} 2 \mathrm{H} / \mathrm{H} 2 \mathrm{M}$ traffic

The remaining part of this section discusses several existing and upcoming issues and challenges posed by M2M communications in LTE/LTE-A networks.

\subsubsection{LTE-A inefficient radio resource utilization}

Existing mobile standards are neither designed to handle small-sized payloads efficiently nor to support large number of devices simultaneously [83]. Consequently, this will lead to network congestion. Current mobile networks must be extended or revised to support massive M2M devices in the future. Since mobile radio resources are valuable assets and scarcely available, it is required to ensure efficient utilization of radio resources for M2M 
communications. According to present 3GPP standardization, the smallest unit of the radio spectrum allocatable to a single device is $1 \mathrm{PRB}$ which is capable to transmit several hundred bits under favorable channel conditions (see TB size for 1 PRB in Fig. 7). However, allocating 1 PRB to a single M2M device could significantly degrade radio spectrum utilization. This is due to the fact that the capacity of a PRB can be much higher than the actual size of the device payload.

Figure 13 illustrates how inefficiently a PRB can be used when a device sends small-sized packets experiencing good channel conditions. Two exemplary device payloads (i.e., 4 and 8 bytes) are considered. Moreover, a PRB utilization efficiency is evaluated in terms of percentage of the actual payload and the padded zeros with respect to the MCS index. It can be seen from Fig. 13 (left) that with the lower MCS values, e.g., 0-6 depicting unfavorable channel conditions, the PRB capacity is smaller, and therefore it is fully utilized to transmit the given payload. On the other hand, with the higher MCS values, e.g., 20-26 representing favorable channel conditions, the capacity of the PRB increases. However, small packets with increasing percentage of padded zeros are transmitted. Similarly, Fig. 13 (right) depicts the percentage of PRB utilization and padded zeros for the second scenario. However, it can be seen from Fig. 13 (right) that for the lower MCS values, the PRB is used efficiently as compared to Fig. 13 (left). However, with the higher values of MCS, the percentage of padded zeros is still significantly large. This shows that approximately 10-20 times more packets can be accommodated per PRB under favorable channel conditions in the given exemplary scenarios.

Due to the inefficient utilization of scarce radio resources, M2M traffic is expected to degrade the performance of traditional mobile traffic. For instance, the authors of $[89,90]$ evaluated the influence of increasing M2M traffic on LTE data traffic through system level simulations using an LTE-A-based model developed in the
OPNET Modeler. The authors concluded that the performance of LTE/LTE-A low priority traffic such as FTP and HTTP is degraded significantly when the number of M2M devices was raised to 750 (Fig. 4 in [89]). This happened due to the fact that e-healthcare traffic was prioritized over LTE traffic.

In LTE/LTE-A networks, radio resources are distributed among several users in order to accomplish user plane and control plane services. The massive number of M2M devices is expected to influence the performance of LTE/LTE-A networks for both the user and the control plane due to the exchange of excessive signaling messages for sending/receiving small packets. Therefore, these topics demand serious attention to enhance M2M services efficiently using LTE/LTE-A networks.

\subsubsection{MTC radio resource allocation mechanisms}

In communication networks, efficient radio resource management has always remained a challenging topic. It has become more challenging for mobile networks to handle traditional traffic co-existing with cellular-based MTC traffic. Therefore, new and optimized radio resource management schemes are required to support future MTC traffic, for which LTE and LTE-A networks are not yet optimized [83]. Several proposals have been presented so far for efficient radio resource allocation for LTE/LTE-A networks.

For instance, authors of [18] proposed two methods for allocating radio resources between MTC and $\mathrm{H} 2 \mathrm{H}$ devices. The first method is based on the orthogonal radio resource allocation. The strength of this resource allocation scheme is its simplicity, whereas the drawback is the low spectral efficiency from the system level aspect. However, the second method, i.e., shared radio resource allocation improves spectral efficiency of the network in a way that MTC devices can reuse the bandwidth allocated to $\mathrm{H} 2 \mathrm{H} / \mathrm{H} 2 \mathrm{M}$ users. However, the drawback is an increased level of interference as compared to the former
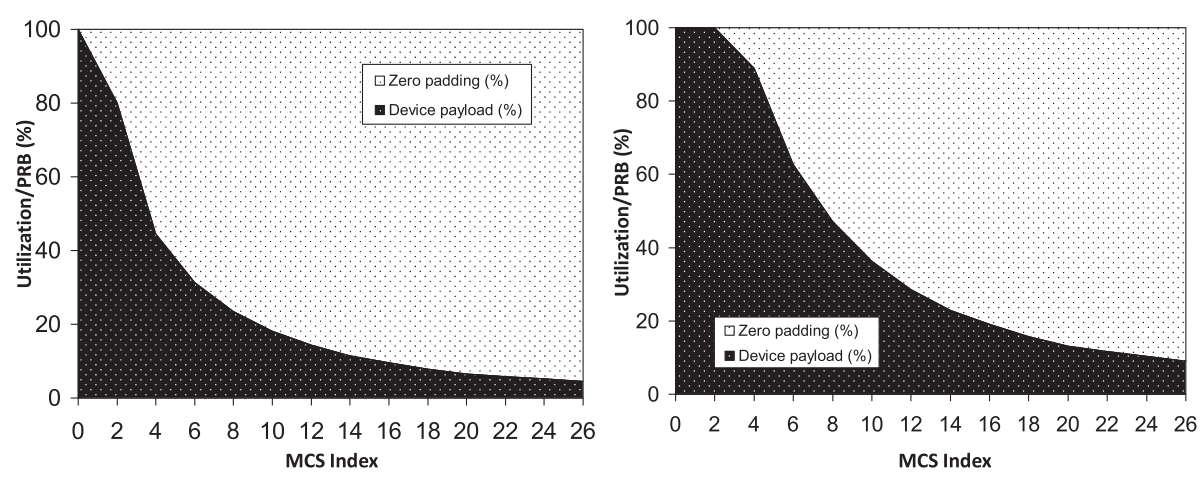

Fig. 13 An illustration of inefficient PRB utilization for M2M communication with device payloads of 4 bytes (left) and 8 bytes (right) 
approach. In 3GPP LTE/LTE-A networks, radio resources are split into PRBs also called subchannels in the context of resource allocation. For instance, if MTC traffic co-exists with traditional $\mathrm{H} 2 \mathrm{H}$ traffic in a communication environment, five distinct links are available which are listed below, see Fig. 14 (taken from [18]):

- $\mathrm{eNB}$ to UE (eNB $\rightarrow$ UE) link

- eNB to MTCD (eNB $\rightarrow$ MTCD) link

- eNB to MTCG (eNB $\rightarrow$ MTCG) link

- MTCG to MTCD (MTCG $\rightarrow$ MTCD) link

- MTCD to MTCD (MTCD $\rightarrow$ MTCD) link

In a mixed traffic environment, one of the major challenges is handling of interference. Therefore, it requires a sophisticated resource partitioning mechanism. The radio resource partitioning enables radio resource allocation among different devices in a coordinated way. The restrictions can be either on the basis of available PRBs or transmit power. As a result, such restrictions provide the possibility for improving the SINR ratio.

Several scheduling algorithms have been proposed for LTE/LTE-A networks. For instance, authors in [91] evaluated the performance of dynamic packet scheduler for LTE networks. The advantage of this approach is the effective control of throughput and fairness between users. However, the drawback of the proposed scheme is that it does not differentiate services. Additionally, authors in [92] introduced a principle of metric decoupling to maximize throughput. Moreover, authors concluded that the fairness among users can be controlled efficiently either through weighting metric in the frequency domain or handling priority in the time domain.
In [93], a path selection criteria for small cells is proposed for joint transmissions on the access and backhaul links in the millimeter wave band to improve the performance of device-to-device (D2D) communication. The proposed D2DMAC scheduling scheme is centralized and outperforms traditional approaches particularly in terms of delay and system throughput. Authors in [94] studied dynamic TDD transmission mechanisms for small cells with several interference management schemes such as interference cancellation, cell clustering, as well as power control. The advantages of proposed schemes are potentially large in low-to-medium traffic loads. Boosting UL power in dynamic TDD scheme significantly outperforms static TDD scheme. Approximately $30-60 \%$ and $210-300 \%$ user throughput is improved in DL and UL, respectively. Moreover, authors in [95] proposed a feasible strategy to enable dynamic TDD transmissions in small cells to achieve UL and DL throughput gains. Additionally, authors in [96] made a theoretical analysis on the SINR performance in case of dynamic time-division duplex (TDD) transmission in small cells. By introducing a partial interference cancellation scheme, outage probabilities are notably decreased. Moreover, authors concluded that the risks of link failures can be significantly reduced by canceling one interfering cell in case of low-to-medium loaded scenarios.

Authors in [97] proposed a cell breathing framework in downlink to minimize breathing effect in femtocells, and thus to balance data rates and cell coverage. A voting-based mechanism, i.e., femtocell virtual election rule (FEVER) is proposed in which users notify the femtocell base station about their current channel conditions which significantly improve system efficiency and fairness among users. In [98], authors studied game dynamics

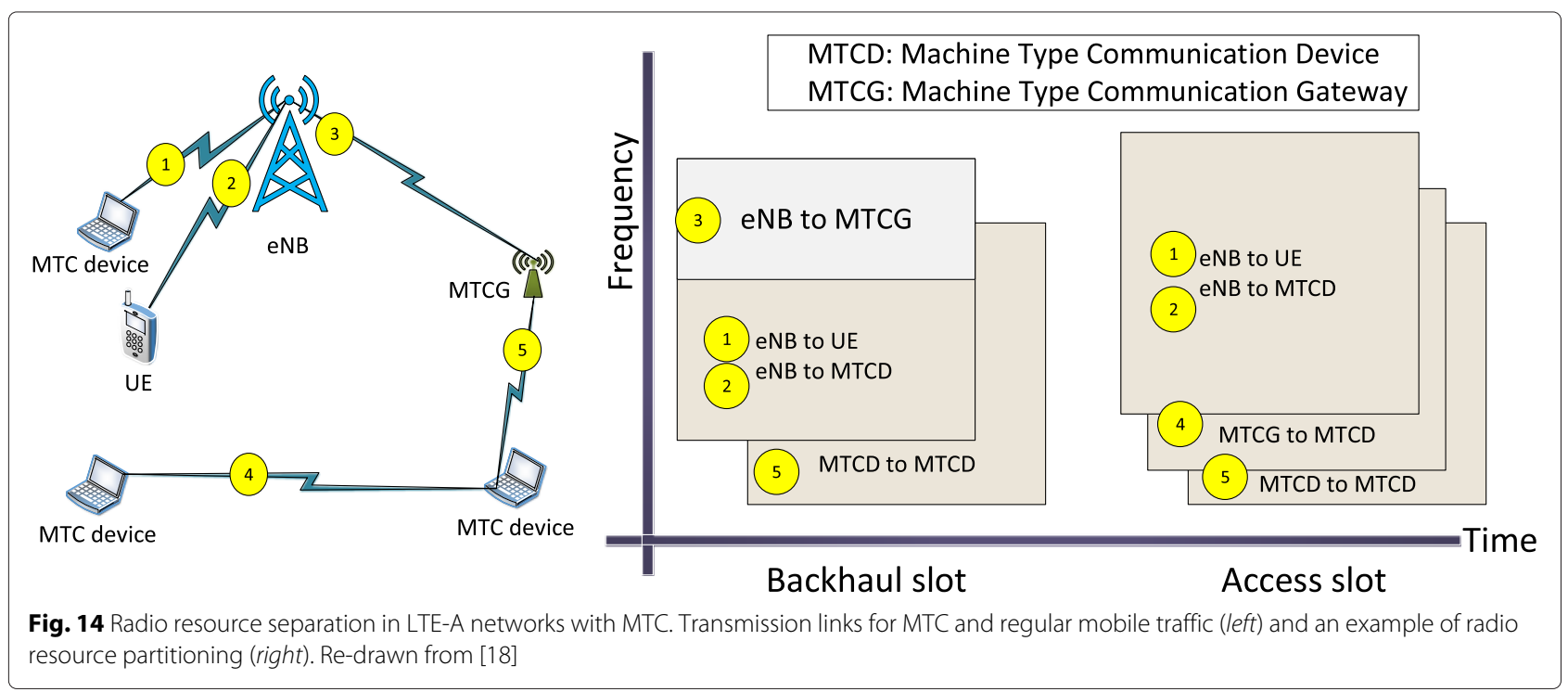


and learning schemes and proposed a novel learning scheme called cost-to-learn which integrates factors such as switching cost and delay as well as user behavior. Resultantly, users concurrently learn their optimal payoff and strategy with the designed CODIPAS-RL (combined fully distributed payoff and strategy reinforcement learning) scheme. Moreover, authors created asymptotic pseudo-trajectories to solve differential equations. In [99], authors proposed a distributed and coordinated scheduling scheme for LTE/LTE-A networks where each cell tends to allocate MCS, PRB, and transmit power to users for minimizing DL power while satisfying user throughput demands. Furthermore, each cell notifies neighboring cells about PRB allocation to cell-edge users. Resultantly, the proposed scheme provides stable solutions for interference mitigation as well as efficient frequency reuse. Moreover, a simple selforganizing scheme is proposed in [100] in which cell transmit power is minimized in order to utilize frequency reuse patterns efficiently in distributed cellular networks.

To support MTC traffic, authors proposed in [101] various scheduling schemes by considering channel conditions as well as delay constraints, thus to maximize the number of supported devices per cell. In [21, 102], authors proposed clustering techniques for MTC traffic. MTC devices are classified into clusters with similar QoS requirements. Fixed AGTI is allocated to each cluster depending on its priority as well as traffic flow. The advantage of the proposed scheme is the capability to support the maximum number of devices while considering their priorities and delay restrictions. The performance of the above mentioned proposals are rather promising at the expense of high signaling overhead [103]. Additionally, there are several limitations in the aforementioned proposals such as the constant MTC traffic flows were examined (contrary to potentially random traffic). In addition, the mean delay experienced by end users is expected as a performance indicator instead of the probabilistic packet delay and loss rate which are recognized as more realistic performance indicators. The authors of [103] attempted to overcome these issues by optimizing the signaling overhead as well as by considering random MTC traffic. The signaling overhead is reduced by merely considering MTC QoS class identifiers (QCIs) which are known in the beginning of a connection and by avoiding feedback messages regarding the buffer status and channel conditions. However, considering various MTC traffic growth forecasts, efficient radio resource management is still one of the most challenging research tasks and demands serious attention [104]. New and advanced schemes are required in order to allocate radio resources efficiently to handle massive MTC traffic in the future.

\subsubsection{M2M random access challenges in LTE- $A$}

In LTE/LTE-A networks, MTC devices contend for PRBs in order to transmit and receive data using an RA procedure. The wireless access schemes can be based either on capillary short-range networks such as WLAN, ZigBee, and IEEE 802.15.4x or long-range mobile networks such as GPRS, GSM, UMTS, and LTE/LTE-A. The wired access techniques are more reliable, introduce small delay, and provide higher throughput. However, it is not suitable for several MTC applications due to various factors such as lack of scalability, cost efficiency, and mobility. In order to cope with these issues, wireless networks play a prominent role. Capillary wireless access is usually used for shortrange links and is less expensive, scalable, and requires low power. However, several factors such as low data rates, weak security, higher interference, and less coverage make it less suitable in most of the MTC applications. In order to provide wider coverage, higher data rates, mobility support, roaming services, and security, mobile networks are more attractive and applicable for future MTC [19]. LTE-A is the latest mobile standard which is a potential option to support MTC. Since MTC devices access the network simultaneously and contend for PRBs, this paper focuses on the contention-based RA challenges and possible proposed solutions.

PRACH overload control Per cell, a massive number of MTC devices is expected to access the network simultaneously [19]. Consequently, it causes a low RA success rate and a high PRACH network congestion. Moreover, unexpected delay, increased packet loss, high power consumption, and inefficient utilization of radio resources will occur. In addition, the PRACH will be further loaded when the increased number of devices will attempt to re-access the network after collisions. Therefore, efficient overload control mechanisms are needed to handle future MTC traffic efficiently.

QoS provisioning In a mixed communication environment, $\mathrm{MTC}$ and $\mathrm{H} 2 \mathrm{H}$ traffic compete for PRACH resources. However, MTC traffic should fulfill its QoS requirements without degrading the performance of traditional $\mathrm{H} 2 \mathrm{H}$ traffic [19]. Since MTC devices are capable to access the eNB either directly or using an intermediate gateway, the decision of selecting an eNB or a gateway can be taken based on the signal strength or channel gain particularly in the case of RA (discussed in Section 1.4.2). In addition, this decision should consider QoS performance based on the contention-based procedure. For instance, a device can receive satisfactory signal strength through direct communications with an eNB. However, if the device uses an intermediate gateway, it can potentially increase RA success probability, reduced power consumption, as well as smaller delay. Furthermore, optimum 
selection of transmission mode should also satisfy MTC QoS requirements such as lower delays, e.g., in the case of emergency/accidental information, and higher data rates, e.g., in surveillance applications. Therefore, the selection of the transmission mode is also crucial. Unfortunately, most of the existing literature does not consider the point selection mechanism for overload control in the RA procedure.

Cognitive M2M communications One of the major issues is the increasing signaling overhead due to the huge number of MTC devices per cell. This increases the possibility of signaling congestion in the network [105]. In addition, there may not be enough bandwidth in order to support and satisfy all devices simultaneously. One of the traditional approaches is to increase the number of eNBs. Furthermore, eNBs supporting MTC and non-MTC traffic should cooperate in order to reduce the risk of network congestion. However, interference between MTC and non-MTC traffic would be a challenging task. Centralized coordination would be effective in order to limit interference. However, it increases the signaling overhead as well as the management complexity. Distributed resource management could be an effective approach in order to limit interference between MTC and non-MTC traffic. Thus, cognitive MTC communication is a promising idea which can be helpful in order to reduce interference between MTC and non-MTC traffic [106]. In addition, the opportunistic random channel mechanisms would be beneficial in order to avoid network congestion. Therefore, enabling cognitive radio capability within an MTC device might be a promising research topic. To handle a large number of devices per cell, random access mechanisms would be beneficial in a way that MTC and non-MTC devices would share the same limited radio spectrum, thus compete for radio resources.

Group-based M2M communication Due to the increasing MTC devices, major challenges are the allocation of RA slots and signaling overhead. In such cases, group-based communication would play a vital role in managing increasing MTC traffic [21]. The primary objective of group-based communication is to reduce the signaling congestion on the air interface. A group head can be selected which collects uplink/downlink data packets, status information, etc. and then forwards the collected information to the corresponding eNB/MTC devices. Since efficient preamble allocation mechanisms are required to avoid collisions, game theory can additionally be a useful tool to optimize preamble allocation [107]. In addition to reduce the network congestion risks, it can also reduce the power consumption of MTC devices. This is due to the fact that machines need to send/receive data to/from a nearby point, e.g., a relay node. In addition, categorizing MTC devices into groups demands special attention as the grouping should be based either on logical or physical characteristics on the basis of, e.g., QoS, jitter, and other MTC traffic characteristics.

\subsubsection{Random access overload control mechanisms}

In order to control overload in LTE-A networks due to massive MTC traffic, the following solutions have been proposed for PRACHs:

Access class barring (ACB) scheme In a traditional $\mathrm{ACB}$ procedure, an eNB broadcasts access probability (AP) and access class (AC) barring time within a cell [19]. When a device initiates the RA mechanism, it draws a number between 0 and 1 . The result of the draw is compared with AP. If the result is less than AP, the device can proceed with the RA mechanism. Otherwise, device has to wait for a duration of AC barring. In Release 10 and onward, 3GPP has proposed an extended access barring (EAB) mechanism in which a certain class of delay tolerant traffic cannot perform the RA procedure whenever an EAB is initiated [108]. The eNB can control the PRACH overload by decreasing the AP value. Resultantly, it lowers the RA attempts. However, it might increase the RA delay for certain devices. Furthermore, in a cooperative ACB mechanism [109], an eNB selects the value of $\mathrm{ACB}$ depending on the level of network congestion. An optimization problem is considered to balance the number of MTC devices attached to a single eNB. However, the drawback of the proposed approach is that it does not consider the priority of MTC devices. In order to cope with this limitation, a priority-aware RA (PRA) scheme is proposed in [110] which takes into account the priority of MTC devices. PRACHs are pre-allocated to multiple MTC classes by adopting a class dependent backoff procedure.

Resource partitioning mechanism The resource partitioning mechanism illustrates the separation of orthogonal PRACH resources/preambles between $\mathrm{H} 2 \mathrm{H}$ and MTC traffic [19]. Two resource partitioning mechanisms are proposed in [111]. In the first method, the preambles are split into two subsets, and each subset is dedicated to MTC and non-MTC devices. In the second method, preambles are again split into two subsets. However, one subset is particularly dedicated to non-MTC traffic, and the second subset is shared by MTC and non-MTC traffic. Once the ACB mechanism is passed successfully, the devices can send preambles using RA. However, the drawback of the proposed approach is that it neglects the eNB selection method and backoff procedure. 
Slotted access scheme In this scheme, each MTC device receives dedicated access slots to perform the RA mechanism and, other than this, always remains in sleep mode [108]. Using ID and RA cycle number, each device can calculate its allowable access slots. An eNB broadcasts the RA cycle number which is a multiple of radio frames. The advantage of the given scheme is that MTC devices can share the access slot if the total number of unique access slots is less than the number of devices within a cell. However, the drawback of this scheme is the large RA request delays if the RA cycle length is increased. However, it can significantly reduce the collisions at the cost of large RA request delays. In the LTE-A RA procedure, the influence of an increasing number of transmission attempts on throughput and delay of the slotted ALOHA-based preamble contention is also studied in [112].

Clustering mechanism for MTC In this scheme, each device can be associated particularly with one or more groups. The radio resources are allocated based on the grouping principle, e.g., MTC devices are grouped based on their QoS requirements [21]. Furthermore, grouping can also be performed based on applications or geographical location. In each group, a group head is selected which communicates with an eNB by relaying requests of the group members to an eNB (i.e., peer-to-peer communication). One of the primary advantages of this scheme is to minimize power consumption for MTC devices [113]. Moreover, it significantly reduces the signaling overhead and ultimately minimizes the risk of network congestion.

Dynamic radio resource allocation In this scheme, an eNB allocates PRACH resources among MTC devices on the basis of PRACH overload and overall traffic load in the network [19]. In a PRACH subframe, a part of the subframe is used by the eNB for performing the PRACH mechanism in order to satisfy QoS and delay requirements, and therefore cannot be used for data transmission. Authors of [114] formulated this issue by reducing the number of PRACH subframes. The expected RA delay is kept within a given delay limit. In addition, a self-optimizing algorithm is proposed in [115] based on the 3GPP proposed slotted access scheme. In the proposed approach, an eNB can dynamically change RA slots for transmitting preambles by taking into account the actual traffic load. The advantage of the proposed scheme is that it minimizes the risk of network congestion.

MTC-specific backoff scheme In this scheme, the MTC and $\mathrm{H} 2 \mathrm{H}$ RA attempts are delayed separately in a way that a smaller value of backoff time, e.g., $20 \mathrm{~ms}$, is given to $\mathrm{H} 2 \mathrm{H}$ traffic and a larger value, e.g., $960 \mathrm{~ms}$, is assigned to MTC traffic [21]. Consequently, this scheme is effective in low overload situations. Whereas in high overload situations, i.e., when more devices perform the RA mechanism simultaneously, this scheme is unable to tackle the network congestion issues. Moreover, the drawback of this proposed scheme is that it does not takes into account the priority of MTC traffic when co-existing with $\mathrm{H} 2 \mathrm{H}$ traffic.

Pull-based scheme This centralized scheme handles situations when more devices attempt to access the network using the RA procedure [108]. In this scheme, an MTC server communicates with an eNB and requests to page MTC devices. When an MTC device receives a paging message from the eNB, it will initiate the RA procedure. Based on the PRACH load and spectrum availability, an eNB is capable to control the required number of MTC devices to be paged. However, the drawback of this scheme is the use of extra control channels to page a massive number of devices.

\subsubsection{QoS provisioning for M2M communications}

QoS provisioning is one of the major requirements in telecommunication systems. In the perspective of service provisioning, ITU-T defines QoS as a set of all servicerelated tasks associated with the service provider when a particular service is offered to the end user [116]. Additionally, QoS parameters are mainly used to evaluate whether or not technologies and offered services fulfill the expectations of end users in terms of quality, reliability, and availability [117].

Unlike traditional mobile traffic, QoS class categorization for M2M communications has remained one of the most challenging tasks for operators due to the fact that M2M applications cover a wide variety of use cases. Since mobile networks are expected as a strong candidate to support future M2M communications, there is a strong requirement for defining unique QoS class categorizations for differentiating M2M traffic, e.g., based on priority and delay. Nevertheless, a particular QoS class categorization for M2M traffic has not yet been defined in the literature [117]. Authors of [118] proposed various M2M QoS classes based on the traffic characteristics. On the other hand, 3GPP and ETSI are putting efforts into allocating specific QCI values to each category in M2M communications. In this survey, several M2M characteristics and traffic models are summarized in Table 8 with a provisional QoS categorization depending on the application type, data packet size, required priority, expected delay, and mobility demands. For instance, it can be seen from Table 8 that M2M devices mainly transmit small packets with an expected inter-arrival time distribution ranging from milliseconds to hours. Furthermore, M2M traffic 
Table 8 Existing QoS class categorization for M2M communications [118, 142, 169]

\begin{tabular}{|c|c|c|c|c|c|}
\hline Parameters & Class 0 & Class 1 & Class 2 & Class 3 & Class 4 \\
\hline Use cases & $\begin{array}{l}\text { e-healthcare, } \\
\text { emergency } \\
\text { alerting }\end{array}$ & $\begin{array}{l}\text { Mobile POS } \\
\text { (point of sale) }\end{array}$ & $\begin{array}{l}\text { Intelligent } \\
\text { transportation, } \\
\text { streaming, }\end{array}$ & $\begin{array}{l}\text { Smart homes, } \\
\text { security }\end{array}$ & $\begin{array}{l}\text { Metering, } \\
\text { monitoring }\end{array}$ \\
\hline Traffic types & $\begin{array}{l}\text { Random, } \\
\text { real time }\end{array}$ & $\begin{array}{l}\text { Random, } \\
\text { real time }\end{array}$ & Random & Random & Regular \\
\hline Priority & Strict, high & Low-1 & Low-2 & Low-3 & Low-3 \\
\hline Data size in bytes & Small & Small & Small & Small/medium & Large \\
\hline Inter-send time & Seconds & Seconds & Minute & $\begin{array}{l}\text { Minute } \\
\downarrow \\
\text { hours }\end{array}$ & $\begin{array}{l}\text { Seconds } \\
\downarrow \\
\text { hours }\end{array}$ \\
\hline Mobility & Low & Low & $\begin{array}{l}\text { Low } \\
\downarrow \\
\text { high }\end{array}$ & No & Low \\
\hline Delay budget & $\begin{array}{l}\text { Milliseconds } \\
\text { [approx. } 125 \text { ms] }\end{array}$ & Milliseconds & $\begin{array}{l}\text { Milliseconds } \\
\downarrow \\
\text { minute }\end{array}$ & Minute & $\begin{array}{l}\text { Seconds } \\
\downarrow \\
\text { hours }\end{array}$ \\
\hline
\end{tabular}

can be classified based on the type of traffic, e.g., real or non-real time traffic. Table 8 depicts various M2M traffic features which can be used to classify M2M traffic into various classes. For instance, emergency alerting, i.e., accidental and/or critical e-healthcare information, is a real-time application which is delay sensitive and therefore demands strict priority. On the other hand, traffic generated by POS terminals is also real time; however, it demands low priority compared to the emergency alerting. In addition, smart metering and monitoring applications generate regular traffic patterns, therefore it can be considered as low priority traffic, but with higher data rate requirements.

\subsubsection{Low-cost low-power device requirements}

In mobile $\mathrm{M} 2 \mathrm{M}$ communications, the cost and power consumption of an M2M module are of significant importance to draw a comparison between cellularbased and non-cellular-based M2M communication services [6]. Unlike traditional mobile data traffic such as VoIP and multimedia services which demand high mobility and have no firm limitations for price and battery time of a device, M2M devices demand low price and low power consumption. 3GPP has considered this issue in its Release 12 and introduces a lowcost M2M device category called Cat-0 device. The average price of this newly introduced Cat- 0 device is approximately $40-50 \%$ of traditional LTE devices. The complexity of the upcoming MTC modem in 3GPP
Release 13 is expected to be $25 \%$ compared to a Cat1 modem. Furthermore, this new category device called Sub Cat-O will not support Tx diversity and MIMO operations. Moreover, narrowband RF design supporting $1.4-\mathrm{MHz}$ bandwidth, data rates up to $200 \mathrm{~kb} / \mathrm{s}$ and transmit power of $20 \mathrm{dBm}$ would sufficiently fulfill MTC application requirements, thus will further reduce device complexity. The key reductions agreed in 3GPP Release 12 and upcoming Release 13 are summarized in Table 9.

In $\mathrm{M} 2 \mathrm{M}$ communications, devices have strict battery power constraints peculiarly in battery-driven M2M devices [6]. Moreover, it is inconvenient to provide services in locations without a direct energy source such as water meters, sensors used in intelligent containers, on vehicles for on-board security, on the body of animals for wildlife management, massive number of metering devices deployed, e.g., in a tower. In such cases, it is expected that M2M devices should work for a longer time period (several years) which is indeed a challenging task for module manufacturers as well as service providers. Furthermore, M2M devices spend most of the time in the idle state due to comparatively longer inter-arrival times. The idle state is designed as a low power state in the LTE network in which devices usually sleep to save battery and wake up periodically to check any system information (SI) update or DL packet arrival by listening to the paging message broadcast by the LTE network [119]. In the existing LTE standards, the maximum allowed paging cycle is 
Table 9 Optimizations of low-cost and low-power M2M devices in 3GPP Release 12 and upcoming Release 13 [6, 170-172]

\begin{tabular}{|c|c|c|c|c|}
\hline Parameters & $\begin{array}{l}\text { Release } 8 \\
\text { (cat-4) }\end{array}$ & $\begin{array}{l}\text { Release } 8 \\
\text { (cat-1) }\end{array}$ & $\begin{array}{l}\text { Release } 12 \\
\text { (cat-0) }\end{array}$ & Release 13 \\
\hline MTC device bandwidth (MHz) & 20 & 20 & 20 & 1.4 \\
\hline Peak data rate downlink & $150 \mathrm{Mbps}$ & $10 \mathrm{Mbps}$ & $1 \mathrm{Mbps}$ & $\begin{array}{l}200 \text { Kbps with a transport } \\
\text { block size (TBS) of } 1000 \text { bits } \\
\text { unicast traffic }\end{array}$ \\
\hline Peak data rate uplink & $50 \mathrm{Mbps}$ & $5 \mathrm{Mbps}$ & $1 \mathrm{Mbps}$ & $\begin{array}{l}200 \text { Kbps with a transport } \\
\text { block size (TBS) of } 1000 \text { bits } \\
\text { unicast traffic }\end{array}$ \\
\hline Duplex mode & Full duplex (opt.) & Full duplex & Half duplex (opt.) & Half duplex (opt.) \\
\hline Max. device Tx power (dBm) & 23 & 23 & 23 & 20 \\
\hline $\begin{array}{l}\text { Modem complexity (\%) } \\
\text { compared to Cat-1 }\end{array}$ & 125 & 100 & 50 & 25 \\
\hline
\end{tabular}

$2.54 \mathrm{~s}$ [120] which is not optimal for M2M communication due to the fact that the inter-arrival time between traffic sessions might be in the range of seconds to minutes. Therefore, consideration of longer paging cycles are under discussion in 3GPP [121, 122]. However, longer paging cycles can increase the delay of DL packets. Therefore, the range of the paging cycle should be selected carefully to keep a balance between delay and power saving efficiency. Figure 15 summarizes the power consumption in different states of a device.

In order to further improve power consumption for M2M devices, several techniques are being considered by 3 GPP in upcoming Release 13. Since downlink traffic can be delay intolerant, it is not possible to use longer
TAUs. Similarly, power consumption can significantly be improved through coverage extension. This is due to the fact that there is always a trade-off between power consumption and reachability. In addition, Release 13 may address functioning of optimized RRC by optimizing signaling overhead. For instance, a remarkable increase in battery life for two AA-size batteries has been observed from 13 to 111 months by increasing the DRX cycle from $2.56 \mathrm{~s}$ to $2 \mathrm{~min}$. The basic idea to improve battery lifetime is to further reduce the activity of the idle mode over the required level for data transmissions/receptions. Thus, the sensors should not have a regular paging cycle which means that devices should only be in active mode if there is any data to transmit.

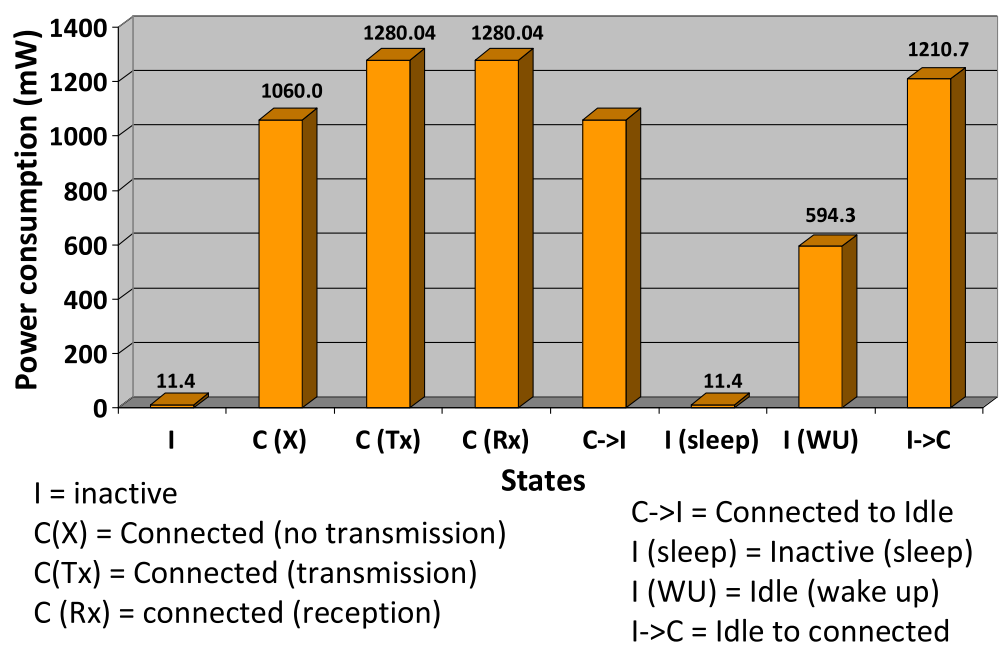

Fig. 15 A comparison of power consumption for different device states [144] 


\subsection{M2M application areas}

Mobile M2M communications cover a broad range of applications, e.g., e-healthcare, transportation and logistics, retail, safety and security, metering and monitoring, automation, and many more. This section discusses major M2M applications which can benefit from mobile networks such as LTE/LTE-A. Moreover, an overview of the major M2M applications covered in this survey is given in Fig. 16.

\subsubsection{Intelligent transportation systems and logistical processes}

Due to the tremendous growth in the number of vehicles, transportation and logistics are considered as two of the main M2M communication domains worldwide [123]. ITS enables the exchange of information between vehicle infrastructure and ITS applications through several communication methods and technologies [5]. In ITS, vehicles communicate with other vehicles (vehicle-to-vehicle $(\mathrm{V} 2 \mathrm{~V})$ ) or with ITS infrastructure server (vehicle-toinfrastructure (V2I)) [50]. The major M2M applications in ITS include collision avoidance, safety and security, parking time management, Internet connectivity, and fuel consumption [3]. In addition, tracking and monitoring of vehicles are the main application areas of mobile M2M communication in transportation and logistics [5, 123]. Several research efforts investigated the support of M2M communications in ITS $[5,124]$. Some of the major M2M applications in ITS are discussed as follows:

Collision avoidance and on-board security Collision avoidance and on-board security are the most significant M2M services in ITS. The main purpose of using mobile M2M communication is to deal with line-of-sight (LoS) limitations in order to avoid accidents [125]. In case of an emergency, the collected information is sent to other vehicles or infrastructure within the communication range. To avoid further incidents, communication between the server and the vehicles must be very fast to detect emergency messages and deliver warning messages immediately. Since the response time to emergency/warning messages is significantly small (i.e., in milliseconds), collision avoidance services demand high QoS and low latency. Furthermore, these warning messages are small in size, thus should only be sent in critical situations to ensure efficient utilization of scarce radio resources in mobile networks [126].

Traffic and infrastructure management All over the world, everyday drivers face the problem of road congestion that not only increases fuel consumption which leads to more emission and causes increased pollution but also causes stress for the drivers [127]. Traffic and infrastructure management can play a significant role in

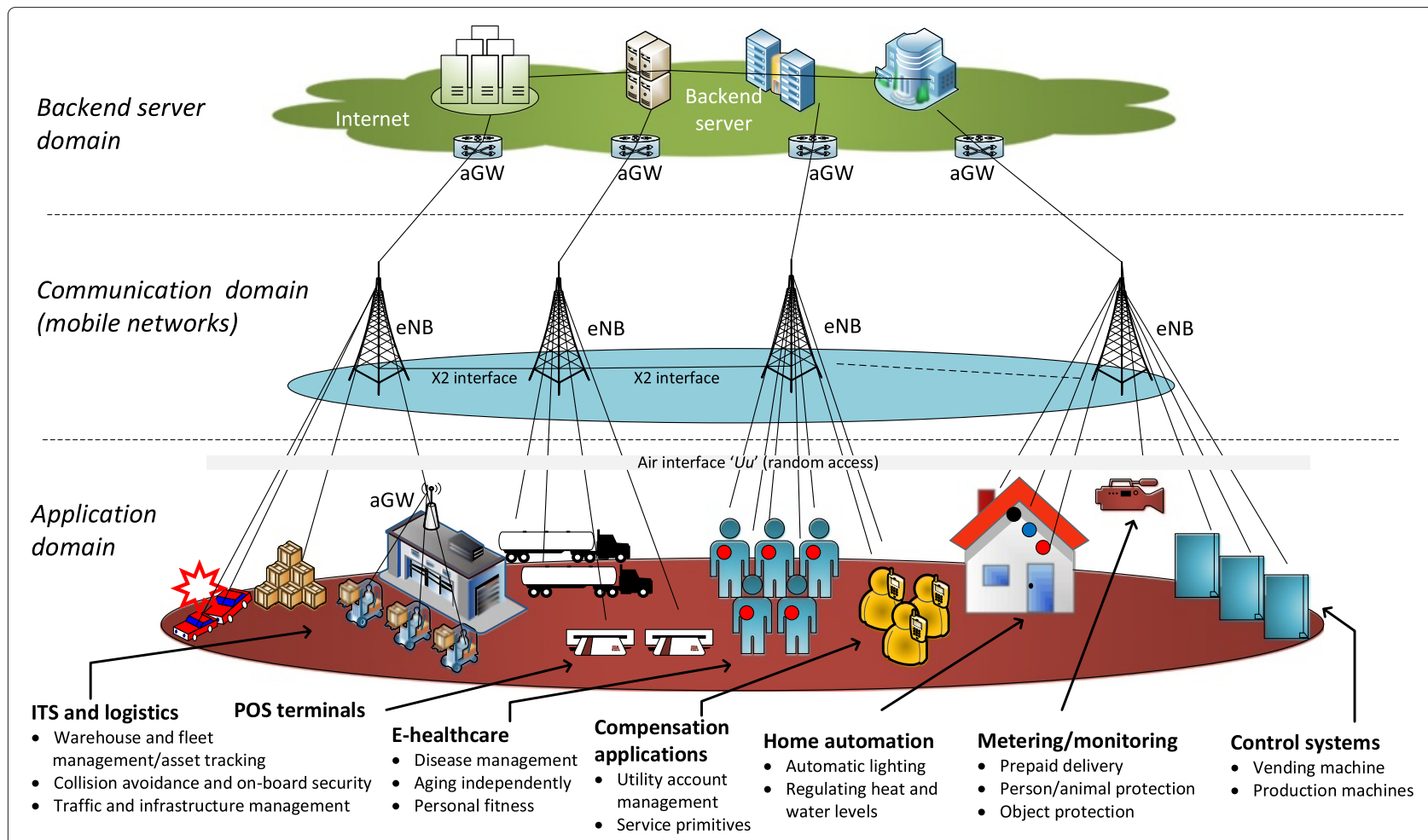

Fig. 16 An overview of mobile M2M applications. Moreover, subcategories of major M2M applications are presented 
handling and controlling the problem of road congestion. ITS tackles the problem by providing bi-directional M2M communications, i.e., between vehicles and ITS server. Vehicles can send status updates about the position, speed, etc. to the infrastructure and can receive information/instructions about road accidents, emergency braking systems etc., from the infrastructure. Therefore, a better managed infrastructure improves the productivity and reduces the factors of cost and pollution in the society. Nevertheless, these applications do not demand high data rates due to the fact that only the abovementioned parameters are mainly sent [128].

The breakdown call (bCall) The bCall service simply starts a voice call and sends the current location/position of a vehicle to an adjacent roadside organization [5]. A bCall service is activated through a triggering operation which is done by the car by pushing triggers. Besides the bCall, an enhanced bCall service provides current diagnostic information along with the location of the vehicle. This allows for a remote diagnosis of faults and enables appropriate and in-time actions to be taken.

Additional M2M services in ITS In addition to the aforementioned applications, M2M communications in ITS support several operations such as tracking of a stolen vehicle, traffic reports and route planning, as well as infotainment services [5]. For instance, to recover a stolen vehicle, stolen vehicle tracking (SVT), service providers request data about the location from Telematic Control Unit (TCU) located inside the vehicle. In addition, drivers are also updated by sending reports regarding traffic in a particular region so that they can change/plan new routes in case of a traffic jam or an emergency. Furthermore, infotainment services aim to provide news/information to drivers and passengers through mobile TV, web-browsing, etc.

M2M in logistical processes Fleet management is one of the major applications of automotive M2M communications in logistical processes [123]. The movements of vehicles, containers, buses, cars, etc. are being tracked regularly through M2M devices which collect data, e.g., of the location, vehicle speed, temperature, distribution progress, fuel consumption, etc., and send this information to monitoring servers. Through regular monitoring, several activities of the system can be performed in an efficient way. For instance, the goods which are transported from one place to another are monitored regularly in order to accomplish in time delivery and to handle any undesirable situation during shipment processes. In summary, M2M communication addresses the following main issues in logistics, (1) Global Connectivity: the cargo moves across several regions/countries and thus must be monitored in order to stay updated; (2) Reporting: with the help of reporting, the exact location of the freight and the conditions of the objects can be easily monitored; and (3) Alarming: in applications such as warehouse management, fleet management, robotics, and control systems alarms are used to detect critical or emergency situations. Besides, M2M communications in logistics can provide additional services such as decreased operational cost, high inventory flexibility, increased supply chain visibility, and reduced loss of vehicles and containers [129].

\subsection{2 e-healthcare}

ITU in 2011 reported the increasing use of M2M services for medicine and electronic health records in e-healthcare [130]. The applications of M2M communications in ehealthcare include, e.g., remote monitoring of patients, provoking of alarms for handling serious conditions, and remote control of certain medical parameters. A patient is equipped with numerous M2M sensors which collect several health indicators of a patient, e.g., blood pressure, temperature, and heart rate. These devices transmit collected data to the monitoring centers such as hospitals, and clinics which store the recorded data and reacts accordingly [69].

Generally, the recorded data is mainly sent to medical centers by using WLAN technology, which is reliable for both the patients and medical control units. However, the lower coverage areas of WLANs may generate problems for the patients in case of mobility outside the home. If the patient is outside the home, e.g., for exercising, the regular monitoring of the patient is not possible due to the limited WLAN coverage. Consequently, in case of any unusual situation, the patient will not be able to get emergency aid by the medical center. In these situations, there is a need to extend the coverage and high mobility support features using mobile networks such as LTE-A. Mobility is one of the fundamental requirements of many M2M applications such as e-healthcare, ITS, and logistics. In this part, some of the major e-healthcare applications are discussed as follows [69]:

Disease management Remote monitoring and management of a disease are two of the common use cases of M2M communications in e-healthcare. Examples of disease management include diabetes management such as tracking the level of blood sugar and regulating the level of the insulin dosage. Furthermore, unusual heartbeats are recorded and sent to the medical center in order to monitor and analyze cardiac arrhythmias. Per time unit, the amount of recorded information as well as the frequency of reporting is mainly dependent on the type of disease to be managed. In addition, alarm functions are ensured in some disease management processes to tackle 
any undesirable situation by sending critical information to the doctor either on regular or on-demand basis.

Independent living with M2M M2M e-healthcare applications allow the people to live independently by monitoring numerous health parameters such as pulse rate, temperature, weight, blood pressure, and sugar level. This also reduces the number of required visits to a medical center. In addition, doctors/care center management can also track whether or not the patients are following the set schedule for taking their medications. Like disease management, sensors acquire data of various indications and then send this to the doctor in order to provide an interface to the patient to receive attention.

Fitness and better health M2M devices are usually deployed to collect/record health concerning parameters such as heartbeats, decreasing rate of fat level, respiratory rate, e.g., during exercise times and jogging. Furthermore, these M2M applications can be used to register several tasks such as duration and frequency of training and exercises. The physician can also make the health profile of a patient by recording data via M2M devices. Furthermore, a doctor/physician can also utilize this information to ensure delivery of precise and quick exercise programs, physiotherapy, etc. and can give comments/feedback to the patients about their recovery, further exercisel plans, etc.

\subsubsection{Smart grid: M2M metering and monitoring services}

Smart metering and monitoring are considered to be one of the main driving forces for increased M2M traffic [131]. Moreover, M2M communications play a vital role for improving the performance of the smart grid which is used to improve the performance of energy generation, distribution, as well as consumption. Smart metering and monitoring are considered to be the major elements used in a smart grid. Devices are deployed, e.g., at the residential area for monitoring of the data, e.g., electricity, gas, water consumption, monitoring and controlling utility consumption, tracing, tracking and protecting people, and assets or animals. Following are the major use cases of smart metering and monitoring [67]:

Prepaid delivery of utilities Companies mainly deploy metering devices which are capable to send information to application servers either automatically or on request. For instance, M2M devices can be used to automatically bill the metering resource. Furthermore, another function of these devices is to improve energy efficiency and performance through accurately measuring readings of the resource consumption, e.g., water, gas, light, etc. Moreover, these applications aim to deliver data of the actual consumption without human intervention. Consequently, it provides and increases a positive environmental impact between service providers and their customers via autonomous communications. In addition, M2M modules can be placed in harsh environments. The limited space available for deploying M2M devices additionally demands small-sized modules. Moreover, the data should be secured and devices should be protected.

Pre-payment is one of the metering applications of gas, electricity, water, etc. With pre-payment, a person/family can buy a particular volume of gas, water, petrol, etc. This information is securely sent and stored within an M2M module. Furthermore, the actual and accurate amount of energy consumed during some particular time is sent to an M2M device. In addition, whenever the household consumes the acquired volume, the actual readings of the used energy are transmitted using an M2M module. When the limit of the purchased volume is reached, warnings in terms of messages or indicators can be sent and the supply can also be stopped.

Person, animal and object protection In these applications, the position as well as status of the person, animal and/or object are monitored. Portable M2M devices with communication modules and an optional GPS functionality are positioned on the body of a person, animal, and/or object. The collected data is sent to the monitoring/caring organizations either autonomously or on demand. The primary objective of these services is to improve the security of people, animals and objects through regular monitoring. Regarding the implementation part, these services are implemented through various applications running within the M2M device. Moreover, portable devices might undergo severe vibrations and shocks as these devices can be deployed in harsh and congested environments. Thus, the device size should be kept small enough to be placed in such an environment easily. In addition, protection of devices must be ensured to avoid cases of theft and misuse, as these devices store sensitive information.

\subsubsection{POS terminals}

Most of the existing POS terminals are connected through wired networks, and therefore fixed at some certain positions, e.g., in shopping malls, restaurants, super markets, railway stations, and airports. If a person is interested to use a POS terminal, then he has to visit the POS terminal. This might cause inconveniences for the users [67]. On the other hand, remotely located POS terminals (e.g., ticketing machines, and parking meters) require a wired connection which is not cost efficient and also difficult to be installed. In addition, this may also be exposed to damages. The other option is to take advantage of 
mobile networks to connect POS terminals. However, this approach poses some security limitations. Introducing M2M devices enable additional possibilities for applications. For instance, online transaction with credit/debit cards and secure communication can be offered with the help of M2M devices which can be deployed in, e.g., parking, stations, airports, and e-ticketing terminals. Typically, these devices have to fulfill several security requirements, e.g., for financial transactions.

\subsubsection{M2M in control systems}

$\mathrm{M} 2 \mathrm{M}$ services provide improved intelligence in industrial plants, robots, furnaces, etc. Sensors and actuators are deployed which can collect and send information to the control room. For instance, if an emergency signal is detected by the sensors, it can be sent immediately to the relevant server. As a result, immediate remedial actions can be taken. Some of the major M2M applications in control systems are discussed below:

Controlling vending machines Due to the increasing product cost, diverse customer choice, increased demand for stock availability, and advanced regulations make a strong competition between operators of vending machines [132]. Vending machines are generally placed inside train stations, offices, public buildings, tourist spots, etc. Nowadays, people are recruited and dedicated to the maintenance of these vending machines [67]. They regularly visit the vending machines to perform several tasks such as checking the fill levels, re-fill if required, maintenance, as well as repairing damages. With the help of M2M technology, additional optimization possibilities are feasible, as a result vending machines can be managed and monitored in an efficient manner. By using devices connected with a mobile network, the current status such as details of the current stock, frequency of customers, damages, and malfunctions can be sent to monitoring centers, e.g., a quality control and maintenance team. In addition, devices can also send updates, e.g., about price variations and thus perform remote maintenance.

Controlling production machines Such machines are usually located inside the production sites which can be a harsh environment for production machines [67]. Dedicated people are hired to perform various tasks related to production machines, e.g., repair and maintenance. They visit production machines on a regular basis and perform several assignments such as repairing damages. The improved performance and maintenance of production machines can be achieved with the use of M2M communications. Thus, machines would be able to communicate with each other, and subsequently send updates to the respective control room. This can significantly increase visibility of the performance of production machines. Besides, in the case of damages or malfunctions, quick identification, and repairing tasks can be performed.

\subsubsection{M2M compensation applications}

$\mathrm{M} 2 \mathrm{M}$ services in compensation applications include the following:

Utility account management A customer will be able to receive an alert about his prepaid account in the case, e.g., when his prepaid account is expected to be finished soon [133]. Thus, he is expected to recharge his prepaid account, e.g., using online banking, and a mobile phone. Furthermore, a trusted third party such as a bank or a credit card company is usually involved in order to perform the verification as well as account recharging tasks. Furthermore, in the case of transportation and logistics, a driver can list, schedule, and deliver goods. Additionally, payments of the delivered goods can also be received electronically. Besides, enterprise POS (EPOS) applications and near-field communication (NFC) payments can be made by benefiting from the compensation capabilities.

Service primitives In management, agreements and remunerations are the basic elements which each company has to deal with. ETSI in its Technical Specification TS 102689 [133] specifies certain high level functions such as CommitValue and Settle which can be used to make agreements between two or more objects/entities. In addition, it is a prerequisite for the agreement that the object must have a legal account as well as relations with the trusted third party such as a bank or a credit card company.

\subsubsection{M2M in home automation}

M2M communications play a vital role in home automation to improve energy consumption, security, etc $[134,135]$. One of the major focuses of home automation with M2M devices is to enhance energy consumption through regular metering and monitoring. For example, M2M devices can be deployed in a room to know about the presence of an individual. In the case, if there is no person present, the lights can be automatically switched off to save energy and ultimately to reduce costs. In addition, water and gas meters can be monitored and regulated using M2M devices. This will significantly improve energy consumption by increasing or decreasing the level of heat or water, e.g., in a room, office, stations, and shopping malls. M2M devices can be connected wirelessly to a gateway or directly to an eNB. Furthermore, the notion of heterogeneous technologies can also be used in such 
applications. It can receive data, merge with the other context information from other devices and send instructions to corresponding actuators.

Mobile M2M devices must use low-power consuming technologies in order to increase their lifetime. Consequently, the customer does not need to change batteries repeatedly. This is also needed for practical reasons, e.g., when M2M devices are installed in some places which are very difficult to approach. These applications can additionally help to inform customers about their energy consumption on a regular basis. This can be achieved with remote monitoring services, i.e., a customer/user can know about the amount of energy consumption even when the user is away from his home. This can also help to notify the user of any detected peculiarity (e.g., leakage of a gas). Consequently, immediate safety measures can be adopted.

\subsection{What is coming next: MTC in 5G}

The race towards developing next generation $5 \mathrm{G}$ networks has started. As a result an increasing interest is shown by several operators, automotive industry, as well as academia to deliver a general framework for 5G. One of the motivations behind the increasing interest in 5G is the expected thousand-fold rise in mobile traffic in 2020 compared to 2010 [136]. METIS is one of the first efforts worldwide to address the requirements for and expectations from this new generation 5G networks [82]. Furthermore, METIS aims to address upcoming challenges due to massive mobile traffic expected in 2020. The progress is achieved in several levels. Firstly, METIS particularly discusses five major 5G scenarios which include "Amazingly fast," "Great service in a crowd," "Ubiquitous things communicating," "Best experience follows you," and "Super real-time and reliable connections" [86, 137]. Furthermore, the major $5 \mathrm{G}$ objectives which are highlighted in the METIS project are given below [86]:

- Per area, the data volume is expected to increase by 1000 times.

- The number of supported devices is believed to be increased by 10-100 times.

- User data rates are anticipated to be increased by 10-100 times.

- In MTC devices with reduced power consumption, the battery life is expected to be increased by ten times.

- E2E delay is envisaged to be reduced by five times.

Supporting future MTC traffic is one of the major focuses of upcoming $5 \mathrm{G}$ networks [86]. In addition, 5G networks are expected to offer improved services in terms of data rates, latency, coverage, users/devices per cell, as well as power consumption for regular UEs and MTC devices. For these reasons, 5G networks are considered to be highly flexible and adaptive to cater the upcoming immense changes in the future. In addition to the aforementioned features, 5G networks are expected to provide high reliability and enhanced the quality of user experience (QoE) for both MTC and non-MTC traffic, therefore METIS has envisaged a user centric 5G network with multi-RAT ${ }^{5}$ capabilities. In summary, the following services are expected for MTC in 5G networks and are depicted in Fig. 17 [137]:

1. $x M B B$ (extreme mobile broadband): $x M B B$ is capable to provide high data rate and at the same time improved QoE through moderate data rate provisioning. It can be seen from Fig. 17 that xMBB is stretched from gigabits per second to megabits per second with increasing reliability. This means that the $\mathrm{xMBB}$ provides high reliability for moderate data rates.

2. MMTC (massive MTC): The focus of $5 \mathrm{G}$ networks is to support low-cost and low-powered MTC devices used in several applications as well as co-exist with conventional non-MTC traffic. mMTC service is anticipated to support massive MTC devices with the reduced date rates when the number of connected sensors increases, as depicted in Fig. 17. Therefore, mMTC emphasizes to support a large number of devices.

3. uMTC (ultra-reliable MTC): 5G networks particularly focus MTC applications such as V2V, V2I, as well as industrial applications. Furthermore, these applications are expected to demand ultra-reliable and time critical services. Therefore, such applications demand high reliability. More importantly, services related to V2V/V2I

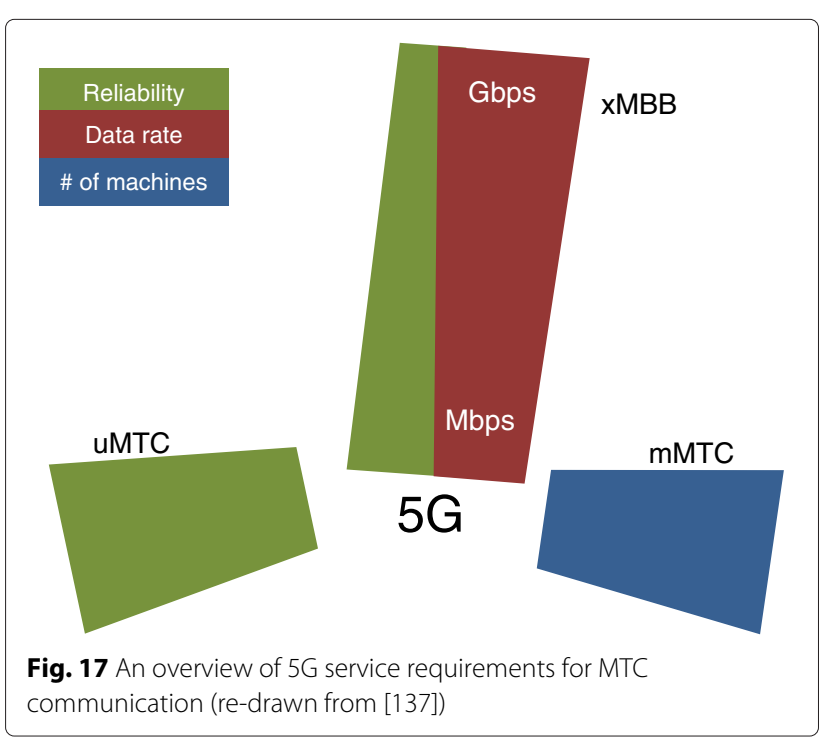


communications demand additional fast communications in order to sense, deliver, receive, and react immediately. Therefore, uMTC emphasizes to support communication with increased reliability, as shown in Fig. 17.

\subsection{Open research areas}

Most of the present M2M-related issues demand either modifications of the contemporary network architecture or standardization of a new network architecture especially for the RAN, to support future MTC in 3GPP systems [17]. Several operators as well as vendors are submitting proposals to 3GPP for the upcoming Release 13 and 14. Huawei along with Qualcomm and other major operators propose for a new network architecture to support mobile MTC. However, others such as Ericsson and Nokia are focusing to modify existing LTE network to support future MTC. Therefore, the system designers have to make sure whether an evolutionary or a revolutionary approach would be more effective for integrating future M2M traffic in mobile networks. In addition, from the perspective of standardization bodies, MTC enhancement tasks are ongoing in 3GPP Release 12 and upcoming Release 13 for optimizing mobile networks to serve MTC traffic. In addition, ETSI, IEEE, and other standardization organizations together with researchers from academia are putting efforts into developing and proposing standards for ensuring MTC communications efficiently. In this section, some of the following principal research issues which demand serious attention are discussed:

\subsubsection{M2M traffic modeling}

Due to the distinctive characteristics of M2M traffic, modeling the behavior of different kinds of M2M applications is one of the major requirements of M2M communications in the future. One of the possibilities is modeling each M2M device on its own. However, there are several challenges that need to be overcome. For instance, it is very difficult to model the behavior of traffic being generated from a massive amount of devices in parallel, and also it becomes more difficult in the presence of strong spatial and temporal correlation between devices [25]. On the other hand, aggregated traffic modeling defines M2M traffic as one stream from multiple devices. Nevertheless, the aggregated traffic models demand additional intermediate gateways between devices and eNBs. In [25], a coupled Markov modulated Poisson process (CMPP) framework was introduced, mainly targeting the source traffic modeling in M2M networks. Furthermore, the authors of [138] introduced the Beta/M/1 model for $\mathrm{M} 2 \mathrm{M}$ communication considering beta-distributed interarrival time for the massive M2M traffic. However, at the same time, the authors stated that a G/M/1 queuing system may be more appropriate to model the behavior of different kinds of bursty applications in M2M communication. In addition, it was shown in [139], that Markov modulated Poisson process (MMPP) models are difficult to fit because they have a large number of parameters. Therefore, it is very difficult to solve them analytically. Since M2M traffic mainly exhibits periodic characteristics, stochastic models, e.g., the one given in [140] for modeling periodic traffic in an IP based system, can be developed to analyze the behavior of M2M traffic. Nevertheless, until now, there has been very little effort towards selecting the most suitable queueing models that can be used to represent the behavior of M2M traffic. In the future, there is a strong need for developing appropriate traffic models for the better understanding of M2M traffic characteristics.

\subsubsection{Random access mechanism}

As discussed in Subsection 1.5.3, the density of M2M devices per cell is expected to be enormous. Therefore, when these devices will try to access the network concurrently, this will lead to a low RA success rate and high network congestion in the PRACH. Consequently, this may cause unexpected delays, increasing packet loss, inefficient radio resource utilization, extra energy consumption as well as service intervention. Moreover, the random access channel can be further overloaded when these devices repeat their attempts to access the network after collisions. Thus, efficient access methods and overload control mechanisms are required to perform efficient RA procedures. Additionally, the initial access procedure in LTE-A networks consists of numerous steps which cause a significant amount of overhead and latency. Authors in [17] further stated that the state-of-the-art access procedures are not appropriate for M2M traffic. Either evolutionary (simpler) access schemes should be standardized for M2M communications or a revolutionary approach, i.e., fully separated standard LTE- and M2M-based data and control channels are required.

\subsubsection{M2M battery life modeling}

Power consumption of an M2M device is of significant importance to compare cellular- and non-cellular-assisted M2M services. Particularly, battery driven M2M devices demand low power consumption to increase their operating (life) time (as discussed in Subsection 1.5.6). Since M2M services are expected to be one of the potential sources to increase revenue of operators, it is important for module manufactures and service providers to design and ensure efficient mechanisms to reduce power consumption for M2M devices. One of the possible approaches can be that of investigating existing battery life models for UEs as well as M2M devices and designing 
an optimized battery life model based on the existing models and by considering more realistic M2M traffic characteristics.

\subsubsection{M2M battery life optimization}

Besides battery life modeling, optimized power consumption mechanisms can significantly increase operating time of an M2M device. For instance, extending sleep mode as well as longer TAUs can be effective to ensure longer battery lifetime. Moreover, optimized RRC, i.e., by optimizing signaling overhead can also be an efficient way to improve power consumption. In general, reducing idle mode activity can also be significantly helpful to improve battery lifetime.

\subsubsection{Efficient PRB utilization}

Existing mobile standards are particularly designed to support broadband traffic. Therefore, LTE TB sizes are only optimized for typical VoIP traffic. These mobile standards must be revised in order to support the enormous $\mathrm{M} 2 \mathrm{M}$ traffic in the future. As mobile radio resources are a valuable asset, these limited radio resources must be efficiently utilized. Since the capacity of a PRB can be significantly larger than the need of typical M2M application (i.e., under favorable channel conditions), allocating 1 PRB to a single M2M device can notably degrade radio spectrum utilization. Hence, alternative schemes such as data aggregation and multiplexing are required for M2M communication. Aggregation and multiplexing of small M2M packets can be achieved by using an intermediate node between devices and eNB. Since relay nodes are one of the latest features of LTE-A networks, they can be used to aggregate traffic from multiple devices. In this way, small packets from different M2M applications can be added to make a comparatively larger packet to maximize radio resource utilization. Besides, it can significantly reduce the risk of network congestion due to the fact that not all devices in the vicinity of the relay node but only a relay node will communicate with the eNB.

\section{Conclusions}

LTE-A and upcoming 5G networks have particularly focused to dominate non-cellular technologies to support future M2M. Therefore, LTE-A is regarded as the readyto-use technology for mobile M2M communication. One of the major challenges that mobile M2M poses is to support the myriad of devices sending small payloads, thus inefficiently utilizing PRBs, causing excessive signaling overhead, and increasing the risk of network congestion. Furthermore, provisioning of low-cost modules (up to US\$10) and low-power devices is another challenge for manufacturers and service providers, respectively. To cater for these issues, schemes such as relaybased data aggregation can be applied to accommodate approximately 10 to 20 times more packets per PRB. Moreover, simpler and opportunistic RA procedures as well as cognitive M2M are candidates to be investigated with respect to reducing signaling overhead and to avoid network congestion. Besides, appropriate traffic models and battery life models need to be developed for future M2M applications.

Techniques for the performance evaluation of the preceding proposals should include all possibilities that are available, e.g., system level simulations, analytical modeling, and hybrid techniques, respectively. The scenarios to be investigated include mainly narrowband applications with small-sized packets, e.g., to evaluate the performance of data aggregation. However, broadband applications need to be part of the traffic mix and can also benefit from some of the changes, e.g., larger TBS. Similarly, the RA procedures are usually complex to model in simulators, thus are candidates to be modeled analytically. Moreover, the performance of simpler and opportunistic RA procedures can be determined and compared with the state-of-the-art RA procedures. Likewise, the performance of optimized power consumption schemes can be assessed through simulations as well as analytically. In addition, the performance of low-power M2M devices in terms of operating time can be determined analytically. Results can also be validated through test bed measurements. More importantly, the major application scenarios to be investigated for low power consumption include deployment of battery-driven devices in locations where there is no or limited direct power source.

Spectrum for mobile networks will remain a scarce resource also in the future and thus new concepts for new traffic types need to be carefully planned and evaluated. Additionally, the IoT will drive new developments including new devices and applications. The future thus poses challenges for all parties involved starting from standardization bodies, manufacturers of network equipment and user devices, network operators, and application providers. In the optimal case, research institutions support these developments by evaluating the new concepts in close cooperation.

\section{Endnotes}

${ }^{1}$ Machine-type communications (MTC) is identical to M2M communication in 3GPP documentations. Since this survey focuses on both ETSI and 3GPP standardization efforts, M2M and MTC terms are used interchangeably in the rest of the paper. Additionally, the terms M2M and MTC are used in the text on the basis of their conventional use by the respective standardization bodies. Moreover, the term non-M2M also called non-MTC represents traditional mobile $\mathrm{H} 2 \mathrm{H}$ and $\mathrm{H} 2 \mathrm{M}$ traffic. 


\section{${ }^{2}$ A bearer is defined as the flow of IP packets between gateways and UEs with a specific QoS. \\ ${ }^{3}$ The SCS represents an entity which is capable to provide value-added services and performs user and control plane communication tasks for MTC [51]. \\ ${ }^{4}$ European Union's Seventh Framework Programme. \\ ${ }^{5}$ Multi random access technologies.}

\section{Competing interests}

The authors declare that they have no competing interests.

\section{Acknowledgments}

We thank the International Graduate School for Dynamics in Logistics (IGS), doctoral training group of LogDynamics, University of Bremen, Germany, and EU programme Erasmus Mundus FUSION (Featured eUrope and South asla mObility Network) Strand 1, Lot 11, Grant Agreement Reference number 2013-2541/001-011 EM Action 2 partnerships, for the financial support of this work.

\section{Author details}

${ }^{1}$ Communication Networks, University of Bremen, Bremen, Germany. ${ }^{2}$ Institute of Communication Networks, University of Technology, Hamburg, Germany.

Received: 22 September 2015 Accepted: 5 November 2015

Published online: 26 November 2015

\section{References}

1. ETSI, Machine-to-machine communications (M2M); Definitions. (ETSI TR 102725 V1.1.1, June, 2013)

2. TTaleb, A Kunz, Machine type communications in 3GPP networks: potential, challenges, and solutions. IEEE Commun. Mag. 50(3), 178-184 (2012)

3. Exalted: Expanding LTE for devices. Available from: http://www.ictexalted.eu/fileadmin/documents/EXALTED_WP2_D2.1.pdf. Accessed 30 Sept 2013

4. Ericsson, Towards 50 billion connected (2010). http://www.ericsson. com/au/res/region_RASO/docs/2010/ericsson_50_billion_paper.pdf. Accessed 10 January 2014

5. ETSI, Machine-to-machine communications (M2M); use cases of Automotive applications in M2M capable networks. (ETSI TR 102898 V1.1.1, April, 2013)

6. NOKIA, LTE M2M: Optimizing LTE for the Internet of Things. (Nokia white paper, 2014). Available from: file:///C:/Users/yasir/Downloads/nokia_ltem_-_optimizing_Ite_for_the_internet_of_things_white_paper.pdf. Accessed 5 August 2015

7. COMARCH, Slicing up the M2M revenue pie: how to get your share and boost your business, (2010)

8. Y Chen, W Wang, in IEEE 72nd Vehicular Technology Conference Fall (VTC 2010-Fall). Machine-to-machine communication in LTE-A (IEEE Ottawa, Canada, 2010), pp. 1-4

9. ERICSSON, ERICSSON Mobility report on the pulse of the networked society, (2014). Available from: http://www.ericsson.com/res/docs/2015/ ericsson-mobility-report-june-2015.pdf. Accessed 10 July 2015

10. 3GPP LTE-Advanced. Available from: http://www.3gpp.org/ technologies/keywords-acronyms/97-Ite-advanced_links.html. Accessed 28 May 2015

11. V Chandrasekhar, JG Andrews, A Gatherer, Femtocell networks: a survey. IEEE Commun. Mag. 46(9), 59-67 (2008)

12. 3GPP, Technical specification group radio access network; evolved universal terrestrial radio access (E-UTRA); relay architectures for E-UTRA (LTE-Advanced) (Release 9). 3GPP TR 36.806 V9.0.0, (2010)

13. C COX, An introduction to LTE: LTE, LTE-Advanced, SAE and $4 G$ mobile communications. (Wiley, New York, 2012)

14. E Kartsakli, AS Lalos, A Antonopoulos, S Tennina, MD Renzo, L Alonso, C Verikoukis, A survey on M2M systems for mHealth: a wireless communications perspective. Sensors. 14(10), 18009-18052 (2014)

15. M Chen, J Wan, F Li, Machine-to-machine communications: architectures, standards and applications. KSII Trans. Internet Inf. Syst. 6(2), 480-497 (2012)
16. K Chen, S Lien, Machine-to-machine communications: technologies and challenges. Ad Hoc Networks (2013). In-Press

17. AG Gotsis, AS Lioumpas, A Alexiou, M2M scheduling over LTE: challenges and new perspectives. IEEE Veh. Technol. Mag. 7(3), 34-39 (2012)

18. K Zheng, F Hu, W Wang, W Xiang, M Dohler, Radio resource allocation in LTE-advanced cellular networks with M2M communications. IEEE Commun. Mag. 50(7), 184-192 (2012)

19. M Hasan, E Hossain, Random access for machine-to-Machine communication in LTE-Advanced networks: issues and approaches. IEEE Commun. Mag. 51(6), 86-93 (2013)

20. ZM Fadlullah, MM Fouda, N Kato, A Takeuchi, N Iwasaki, Y Nozaki, Toward intelligent machine-to-machine communications in smart grid IEEE Commun. Mag. 49(4), 60-65 (2011)

21. S Lien, K Chen, Y Lin, Toward ubiquitous massive accesses in $3 \mathrm{GPP}$ machine-to-machine communications. IEEE Commun. Mag. 49(4), 66-74 (2011)

22. Z Sheng, S Yang, Y Yu, AV Vasilakos, J Mccann, K Leung, A survey on the IETF protocol suite for the internet of things: standards, challenges, and opportunities. IEEE Wirel. Commun. 20(6), 91-98 (2013)

23. M Yang, Y Li, D Jin, L Zeng, X Wu, AV Vasilakos, Software-defined and virtualized future mobile and wireless networks: a survey. Mob. Networks and Appl. 20(1), 4-18 (2014)

24. J Wan, M Chen, F Xia, L Di, K Zhou, From machine-to-machine communications towards cyber-physical systems. Comput. Sci. Inf. Syst. 10(3), 1105-1128 (2013)

25. M Laner, P Svoboda, N Nikaein, M Rupp, in Proceedings of the Tenth International Symposium on Wireless Communication Systems (ISWCS). Traffic Models for Machine Type Communications (VDE IImenau, Germany, 2013), pp. 1-5

26. MZ Shafiq, L Ji, AX Liu, J Pang, J Wang, in ACM SIGMETRICS Performance Evaluation Review. A first look at cellular machine-to-machine traffic: large scale measurement and characterization, vol. 40, (2012), pp. 65-76

27. 3GPP, Technical Specification Group Services and System Aspects; Service accessibility (Release 12). (3GPP TS 22.011 V13.1.0, 2014)

28. ETSI, Machine-to-Machine communications (M2M): Functional architecture. (ETSI TS 102690 V2.1.1, 2010). Available from: http://www. etsi.org/deliver/etsi ts/102600_102699/102690/02.01.01_60/ts 102690v020101p.pdf

29. A Kumar, J Sengupta, Y Liu, 3GPP LTE: the future of mobile broadband. Wirel. Pers. Commun. 62(3), 671-686 (2012)

30. 3GPP, Technical Specification Group Radio Access Network; requirements for evolved UTRA (E-UTRA) and evolved UTRAN (E-UTRAN) (Release 9). (3GPP TR 25.913 V9.0.0, 2009)

31. 3GPP, Technical Specification Group Radio Access Network; evolved universal terrestrial radio access network (E-UTRAN); architecture description (Release 9). (3GPP TS 36.401 V10.0.0, 2010)

32. Innovations T, LTE in a nutshell: protocol architecture (2010). Available from: http://www.tsiwireless.com/docs/whitepapers/LTE. Accessed 2 August 2015

33. A Meylan, 3GPP LTE Radio layer 2, RRC and Radio Access Network Architecture. (3GPP TSG-RAN WG2, 2010). Available from: ftp://www. 3gpp.org/Information/presentations/presentations_2010/2010_06_ India/3GPP\%20LTE\%20Radio\%20layer\%202.pdf. Accessed 13 January 2015

34. LTE E-UTRAN and its access side protocols. White Paper (2010). Available from: http://go.radisys.com/rs/radisys/images/paper-lte-eutran.pdf. Accessed 7 September 2015

35. 3GPP, Technical Specification Group Radio Access Network; evolved universal terrestrial radio access (E-UTRA); radio resource control (RRC); protocol specification (Release 12). (3GPP TS 25.331 V12.6.0, 2015)

36. 3GPP, Technical Specification Group Radio Access Network; evolved universal terrestrial radio access (E-UTRA); packet data convergence protocol (PDCP) specification (Release 12). (3GPP TS 36.323 V12.2.0, 2014)

37. 3GPP, Technical Specification Group Radio Access Network; evolved universal terrestrial radio access (E-UTRA); radio link control (RLC) protocol specification (Release 12). (3GPP TS 36.322 V12.1.1, 2014)

38. 3GPP, Technical Specification Group Radio Access Network; evolved universal terrestrial radio access (E-UTRA); medium access control (MAC) protocol specification (Release 12). (3GPP TS 25.321 V12.2.0, 2014)

39. 3GPP, Technical Specification Group Radio Access Network; evolved universal terrestrial radio access (E-UTRA); LTE physical layer; general description (Release 12). (3GPP TS 36.201 V12.2.0, 2015) 
40. 3GPP, Technical Specification Group Radio Access Network; evolved universal terrestrial radio access (E-UTRA); user equipment (UE) radio transmission and reception (Release 10). (3GPP TS 136.101 V10.3.0, 2011)

41. S Sesia, I Toufik, M Baker, LTE: the UMTS long term evolution. (Wiley Online Library, New York, 2009)

42. 3GPP, Technical Specification Group Radio Access Network. Evolved universal terrestrial radio access (E-UTRA) and evolved universal terrestrial radio access network (E-UTRAN). Overall description. 3GPP TS 36.300 V11.2.0 (2012)

43. 3GPP, Technical Specification Group Radio Access Network. Evolved Universal Terrestrial Radio Access (E-UTRA) and Evolved Universal Terrestrial Radio Access Network (E-UTRAN). Overall description Stage 2 (Release 13). 3GPP TS 36.300 V13.0.0 (2015)

44. 3GPP, Technical Specification Group Radio Access Network; Evolved Universal Terrestrial Radio Access (E-UTRA); Medium Access Control (MAC) protocol specification (Release 12). 3GPP TS 36.321 V12.0.0 (2014)

45. 3GPP, Architecture enhancements to facilitate communications with packet data networks and applications (Release 12). 3GPP TS 23.682 V12.4.0 (2015)

46. 3GPP, Technical Specification Group Radio Access Network; Requirements for further advancements for Evolved Universal Terrestrial Radio Access (E-UTRA)(LTEAdvanced)( Release 12). 3GPP TR 36.913 V12.0.0 (2014)

47. P Mogensen, W Na, IZ Kovács, F Frederiksen, A Pokhariyal, KI Pedersen, K Kolding, K Hugl, M Kuusela, in IEEE 65th on Vehicular Technology Conference. LTE capacity compared to the shannon bound (IEEE Dublin, Ireland, 2007), pp. 1234-1238

48. U Barth, 3GPP Long-Term Evolution/system architecture evolution overview (Alcatel White Paper) (2006). Avilable from: http://www.ikr.unistuttgart.de/Content/itg/fg524/Meetings/2006-09-29-Ulm/01-3GPP_ LTE-SAE_Overview_Sep06.pdf, accessed 19 February 2015

49. ETSI, Machine-to-machine communications (M2M), Functional architecture. (ETSI TS 102.690 V1.1.1, 2011). Avilable from: http://www. etsi.org/deliver/etsi_ts/102600_102699/102690/01.01.01_60/ts_ 102690v010101p.pdf, accessed 15 March 2015

50. MJ Booysen, S Zeadally, GV Rooyen, Survey of media access control protocols for vehicular ad hoc networks. IET Commun. 5(11), 1619-1631 (2011)

51. 3GPP, Architecture enhancements to facilitate communications with packet data networks and applications (Release 13). (3GPP TS 23.682 V13.1.0, 2015)

52. 3GPP, Architecture enhancements to facilitate communications with packet data networks and applications (Release 11). (3GPP TS 23.682 V11.5.0, 2013)

53. 3GPP, Architecture enhancements to facilitate communications with packet data networks and applications (Release 13). (3GPP TS 23.682 V13.2.0, 2015)

54. 3GPP, Service requirements for machine-type communications (MTC) (Release 11). (3GPP TS 22.368 V11.5.0, 2012)

55. 3GPP, Technical Specification Group Services and System Aspects; service requirements for machine-type communications (MTC); Stage 1 (Release 13). (3GPP TS22.368 V13.1.0, 2014)

56. 3GPP, Technical Specification Group Services and System Aspects; Service requirements for machine-type communications (MTC); Stage 1 (Release 10). (3GPP TS 22.368 V10.5.0, 2011)

57. 3GPP, Technical Specification Group Services and System Aspects; Service requirements for machine-type communications (MTC); Stage 1 (Release 11). (3GPP TS 22.368 V11.6.0, 2011)

58. 3GPP, Technical Specification Group Services and System Aspects; Service requirements for machine-type communications (MTC); Stage 1 (Release 12). (3GPP TS 22.368 V12.4.0, 2014)

59. Z Fan, RJ Haines, P Kulkarni, M2M communications for E-health and smart grid: an industry and standard perspective. IEEE Wireless Communications. 21(1), 62-69 (2013)

60. Standards for $\mathrm{M} 2 \mathrm{M}$ and the Internet of Things. Available from: http:// www.onem $2 \mathrm{~m}$.org/. Accessed 28 Aug 2015

61. 3GPP, Study on enhancements for machine type communication (Release 12). (3GPP TR $22.888 \mathrm{~V} 1.0 .0,2013$ )

62. 3GPP, Feasibility study on the security aspects of remote provisioning and change of subscription for machine to machine (M2M) equipment. (TR 33.812 V9.1.0, 2010)

63. 3GPP, Security aspects of machine-type and other mobile data applications communications enhancements (Release 12). (3GPP TR 33.868 V0.10.0, 2012)
64. 3GPP, Standardization of machine-type communications, V0.2.4, (2014) Available from: http://wenku.baidu.com/view/ 163f99d5960590c69ec3767f. Accessed 01 July 2015

65. ETSI, Machine-to-machine communications (M2M); Interworking between the M2M architecture and M2M area network technologies. (ETSI TR 102 966 V1.1.1, 2014)

66. ETSI, Machine-to-machine communications (M2M); mla, dla and mld interfaces. (ETSI TS 102921 V1.1.1, 2012)

67. ETSI, Machine-to-machine communications (M2M); M2M service requirements. (ETSI TS 102689 V1.1.1, 2010)

68. ETSI, Machine-to-machine communications (M2M); Interoperability Test Specification for COAP Binding of ETSI M2M Primitives. (ETSI TS 103104 V2.1.1, 2013)

69. ETSI, Machine-to-machine communications (M2M); use cases of M2M applications for e-health. (ETSI TR 102732 V1.1.1, 2013)

70. ETSI, Machine-to-machine communications (M2M); M2M service requirements. (ETSI TR 102691 V1.1.1, 2010)

71. ETSI, Machine-to-machine communications (M2M); use of automotive applications in M2M capable Networks. (ETSI TR 102898 V1.1.1, 2014)

72. ETSI, Machine-to-machine communications (M2M); applicability of M2M architecture in smart grid networks, impact of smart grid on M2M platform. (ETSI TR 102935 V2.1.1, 2012)

73. IEEE 802 16p-11/0033, Enhancements to support machine-to-machine applications for WirelessMAN-Advanced. (IEEE 802.16's Machine-to-Machine (M2M) Task Group, 2010). Available from: http:// ieee802.org/16/m2m/. Accessed 03 July 2015

74. IEEE 802 16p-10/0004, Machine to machine (M2M) system requirements document (SRD). (IEEE 802.16Šs Machine-to-Machine (M2M) Task Group, 2010). Available from: http://ieee802.org/16/m2m/. Accessed 09 September 2014

75. G Jones, OMA and Machine-to-Machine (M2M) communication (White Paper), (2011). Available from: http://openmobilealliance.org/static/ oma-annual-reports/documents/oma\%20collateral\%20m2m\%205-11. pdf. Accessed 17 August 2015

76. TIA TR-50 framework intelligent device/smart devices. Available from: http://ftp.tiaonline.org/TR-30/TR-30.2/Public/2009\%20Meetings/200909\%20Arlington/20909008b\%20TIA\%20Intelligent\%20Device \%20Persentation.pdf. Accessed 28 Aug 2015

77. Remote SIM Provisioning for machine to machine. Available from: http://www.gsma.com/connectedliving/embedded-sim/. Accessed: 12 June 2015

78. AT4 wireless laboratories announces the first NFC Forum device certification. Available from: http://www.m2mnow.biz/2012/03/31/ 4833-at4-wireless-laboratories-announces-the-first-nfc-forum-devicecertification/. Accessed 20 July 2015

79. Discover Wi Fi. Available from: http://www.wi-fi.org/discover-wi-fi. Accessed 12 June 2015

80. Open Mobile Alliance (OMA). Available from: http://openmobilealliance. org/about-oma/work-program/m2m-enablers/. Accessed 01 Apr 2014

81. China Communications Standards Association. Available from: http:// www.ccsa.org.cn/english/. Accessed 20 July 2015

82. Mobile and Wireless Communications Enablers for Twenty-Twenty (2020). Available from: https://www.metis2020.com/?doing_wp_cron= 14409434915532760620117187500000_links.html. Accessed 28 Aug 2015

83. Mobile and wireless communications Enablers for Twenty-twenty (2020) Information Society, Novel radio link concepts and state of the art analysis. Deliverable Number ICT-317669-METIS/D2.2, 2013. Available from: https://www.metis2020.com/wp-content/uploads/deliverables/ METIS_D2.2_v1.pdf, accessed 12 October 2014

84. Achieving low-latency in wireless communications (LOLA). Available from: http://www.ict-lola.eu/. Accessed 30 June 2015

85. CISCO, Cisco Visual Networking Index: Global Mobile Data Traffic Forecast Update. 2014-2019 (white paper), http://www.cisco.com/c/en/ us/solutions/collateral/service-provider/visual-networking-index-vni/ white_paper_c11-520862.pdf Accessed 20 July 2015

86. Mobile and wireless communications Enablers for Twenty-twenty (2020) Information Society, Scenarios, requirements and KPIs for $5 \mathrm{G}$ mobile and wireless system. (Deliverable Number ICT-317669-METIS/D1.1, 2013). Available from: https://www.metis2020.com/wp-content/uploads/ deliverables/METIS_D1.1_v1.pdf, accessed 12 October 2014 
87. Cellular M2M forecasts: unlocking growth. Available from: https:// gsmaintelligence.com/research/2015/02/cellular-m2m-forecastsunlocking-growth/457//\%20Accessed:\%206\%20Feb\%202015/_links. html. Accessed 23 May 2015

88. Report: M2M device connections forecast 2014-2024. Available from: http://www.machinetomachinemagazine.com/2014/08/20/reportm2m-device-connections-forecast-2014-2024/. Accessed 23 May 2015

89. T Pötsch, SNK Marwat, Y Zaki, C Görg, in IEEE 6th Joint IFIP Wireless and Mobile Networking Conference (WMNC). Influence of Future M2M Communication on the LTE system (IEEE, Dubai, UAE, 2013), pp. 1-4

90. Y Mehmood, SNK Marwat, T Pötsch, F Ahmad, C Görg, I Rashid, Impact of M2M traffic on LTE data traffic performance. (Springer, Bremen, Germany, 2014)

91. P Kela, J Puttonen, N Kolehmainen, T Ristaniemi, T Henttonen, M Moisio, in 3rd International Symposium on Wireless Pervasive Computing. Dynamic packet scheduling performance in UTRA long term evolution downlink (IEEE Santorini, Greece, 2008), pp. 308-313

92. G Monghal, KI Pedersen, PE Mogensen, in IEEE Vehicular Technology Conference. QoS oriented time and frequency domain packet schedulers for the UTRAN long term evolution (IEEE Singapore, 2008), pp. 2532-2536

93. Y Niu, C Gao, Y Li, L Su, D Jin, A Vasilakos, Exploiting Device-to-Device Communications in Joint Scheduling of Access and Backhaul for mmWave Small Cells, (2015). accepted in IEEE Journal on Selected Areas in Communications http://arxiv.org/pdf/1503.02292v1.pdf

94. M Ding, D López-Pérez, AV Vasilakos, W Chen, in IEEE International Conference on Communications Workshops (ICC). Dynamic TDD transmissions in homogeneous small cell networks (IEEE, Sydney, NSW, 2014), pp. 616-621

95. M Ding, D Lopez-Perez, X Ruiqi, AV Vasilakos, W Chen, in IEEE International Conference on Communications (ICC). Small cell dynamic TDD transmissions in heterogeneous networks (IEEE, Sydney, NSW, 2014), pp. 4881-4887

96. M Ding, D López-Pérez, AV Vasilakos, W Chen, in IEEE Global Communications Conference (GLOBECOM). Analysis on the SINR performance of dynamic TDD in homogeneous small cell networks (IEEE, Austin, Texas, 2014), pp. 1552-1558

97. CY Wang, CH Ko, HY Wei, AV Vasilakos, A voting-based femtocell downlink cell-breathing control mechanism. IEEE/ACM Trans. Networking (TON), (2014), p. 99

98. MA Khan, H Tembine, AV Vasilakos, Game dynamics and cost of learning in heterogeneous 4G networks. IEEE J. Sel. Areas Commun. 30(1), 198-213 (2012)

99. D López-Pérez, X Chu, AV Vasilakos, H Claussen, On distributed and coordinated resource allocation for interference mitigation in self-organizing LTE networks. IEEE/ACM Transactions on Networking (TON). 21(4), 1145-1158 (2013)

100. D López-Pérez, X Chu, AV Vasilakos, H Claussen, Power minimization based resource allocation for interference mitigation in OFDMA femtocell networks. IEEE J. Sel. Areas Commun. 32(2), 333-344 (2014)

101. AS Lioumpas, A Alexiou, in IEEE GLOBECOM Workshops (GC workshops). Uplink scheduling for machine-to-machine communications in LTE-based cellular systems (IEEE, Houston, Texas, 2011), pp. 353-357

102. S Lien, K Chen, Massive access management for QoS guarantees in 3GPP machine-to-machine communications. IEEE Commun. Lett. 15(3), $311-313$ (2011)

103. AG Gotsis, AS Lioumpas, A Alexiou, Analytical modelling and performance evaluation of realistic time-controlled M2M scheduling over LTE cellular networks. Trans. Emerg. Telecommun. Technol. 24(4), 378-388 (2013)

104. Y Mehmood, SNK Marwat, Y Zaki, C Görg, A Timm-Giel, in 20 ITG Mobile Communication Conference. Evaluation of M2M Data Traffic Aggregation in LTE-A Uplink (VDE, Osnabrück, Germany, 2015), pp. 24-29

105. F Ghavimi, H Chen, M2M Communications in 3GPP LTE/LTE-A networks: architectures, service requirements, challenges and applications. IEEE Commun. Surv. Tutorials. 17(2), 525-549 (2015)

106. Y Zhang, R Yu, M Nekovee, Y Liu, S Xie, S Gjessing, Cognitive machine-to-machine communications: visions and potentials for the smart grid. IEEE Netw. 26(3), 6-13 (2012)
107. H Safdar, N Fisal, R Ullah, W Maqbool, F Asraf, Z Khalid, AS Khan, in IEEE Symposium on Wireless Technology and Applications (ISWTA). Resource allocation for uplink M2M communication: a game theory approach (IEEE, Kuching, Malaysia, 2013), pp. 48-52

108. 3GPP, Study on RAN Improvements for Machine Type Communications (Release 11). (3GPP TR 37.868 V11.0.0, 2011)

109. S Lien, T T Liau, C Kao, K Chen, Cooperative access class barring for machine-to-machine communications. IEEE Trans. Wirel. Commun. 11(1), 27-32 (2012)

110. J Cheng, C Lee, T Lin, in IEEE GLOBECOM Workshops (GC workshops). Prioritized random access with dynamic access barring for RAN overload in 3GPP LTE-A networks (IEEE, Houston, Texas, 2011), pp. 368-372

111. K Lee, S Kim, B Yi, in IEEE GLOBECOM Workshops (GC Workshops). Throughput comparison of random access methods for M2M service over LTE networks (IEEE, Houston, Texas, 2011), pp. 373-377

112. RR Tyagi, F Aurzada, K Lee, SG Kim, M Reisslein, Impact of retransmission limit on preamble contention in LTE-Advanced network. IEEE Systems Journal. 9(3), 752-765 (2014)

113. C Tu, C Ho, C Huang, in IEEE Vehicular Technology Conference (VTC Fall). Energy-efficient algorithms and evaluations for massive access management in cellular based machine to machine communications (IEEE, Budapest, Hungary, 2011), pp. 1-5

114. S Choi, W Lee, D Kim, K Park, S Choi, K Han, in IEEE IFIP Wireless Days (WD). Automatic configuration of random access channel parameters in LTE systems (IEEE, Niagara Falls, ON, Canada, 2011), pp. 1-6

115. A Lo, YW Law, M Jacobsson, M Kucharzak, in 27th World Wireless Research Forum (WWRF) Meeting. Enhanced LTE-advanced random-access mechanism for massive machine-to-machine (M2M) communications (WWRF27-WG4-08, Düsseldorf, Germany, 2011), pp. 1-5

116. International Telecommunication Union Telecommunications (ITU-T), Definitions of terms related to quality of service (2008). Available from: https://www.itu.int/rec/T-REC-E.800-200809-I/en, accessed 10 March 2015

117. S Zrncic, I Bojic, D Katusic, P Skocir, M Kusek, G Jezic, in 21st IEEE International Conference on Software, Telecommunications and Computer Networks (SoftCOM). Quality-of-service in machine-to-machine service provisioning process (IEEE, Split-Primosten, Croatia, 2013), pp. 1-5

118. R Liu, W Wu, H Zhu, D Yang, in IEEE 7th International Conference on Networking and Mobile Computing (WiCOM). M2M-oriented QoS categorization in cellular network (IET, Beijing, China, 2011), pp. 1-5

119. 3GPP. Evolved Universal Terrestrial Radio Access (EUTRA); User Equipment (UE) procedures in idle mode (Release 11), 3GPP TS 36.304 V11.3.0 (2013)

120. 3GPP. Evolved Universal Terrestrial Radio Access (EUTRA); Radio Resource Control (RRC); Protocol specification (Release 11), 3GPP TS 36.331 V11.3.0, (2013)

121. 3GPP. Service requirements for machine-type communications (MTC); stage 1 (Release 12), 3GPP TS 22.368 V12.2.0, (2013)

122. 3GPP. Service requirements for machine-type communications (MTC); stage 1 (Release 12), 3GPP TS 22.368 V12.2.0 (2013)

123. The state of $M 2 M$ in transport and logistics; Key fidings from the 2013 Vodafone M2M Adoption Barometer (2014). Available from: http://www. automotiveit.eu/wp-content/uploads/2014/07/ Vodafone_M2M_Barometer_2014.pdf, accessed 18 February 2015

124. V Kapsalis, C Fidas, L Hadellis, C Karavasilis, M Galetakis, C Katsenos, in 13th International IEEE Conference on Intelligent Transportation Systems (ITSC). A networking platform for real-time monitoring and rule-based control of transport fleets and transferred goods (IEEE, Funchal, Portugal, 2010), pp. 295-300

125. MJ Booysen, JS Gilmore, S Zeadally, GV Rooyen, Machine-to-machine (M2M) communications in vehicular networks. KSII Trans. Internet Inf. Syst. 6(2), 529-546 (2012)

126. K Bilstrup, E Uhlemann, EG Ström, U Bilstrup, On the ability of the 802.11 p MAC method and STDMA to support real-time vehicle-to-vehicle communications. EURASIP J. Wirel. Commun. Netw. 2009(902414), 1-14 (2009)

127. H Li, in Second IEEE International Conference on Mechanic Automation and Control Engineering (MACE). Calculation of additional pollutant gas emissions and their social cost from transport congestion (IEEE, Wuhan China, 2011), pp. 5639-5641 
128. DAP Greenwood, C Dannegger, K Dorer, M Calisti, in HICSS. Dynamic dispatching and transport optimization-real-world experience with perspectives on pervasive technology integration, (2009), pp. 1-9

129. Machine to machine (M2M) - DEUTSCHE TELEKOM; Transport and Logistics. Available from: https://m2m.telekom.com/vertical-industries/ transport-and-logistics. Accessed 01 Sept 2015

130. International Telecommunication Union Telecommunications (ITU-T), Standards and eHealth ITU-T Technology Watch Report, (2011). Available from: https://www.itu.int/dms_pub/itu-t/oth/23/01/ T23010000170001PDFE.pdf, accessed 19 December 2014

131. Singtel: Machine to machine application for smart metering and utilities. Available from: http://info.singtel.com/large-enterprise/m2m/machineto-machine-application/smart-metering-utilities. Accessed $01 \mathrm{Sept}$ 2015

132. EUROTECH: Vending machine management. Available from: http:// www.eurotech.com/en/industry+solutions/industrial+commercial/ vending+machine. Accessed 20 Aug 2015

133. ETSI. Machine-to-Machine communications (M2M); M2M service requirements, ETSI TS 102689 V1.2.1, (2010)

134. ETSI. Machine-to-Machine communications (M2M); Impact of Smart City Activity on loT Environment, ETSI TR 103290 V1.1.1, (2015)

135. ETSI. Machine-to-machine communications (M2M); use cases of M2M applications for connected consumer, ETSI TR 102857 V1.1.1, (2013)

136. A Osseiran, V Braun, THidekazu, P Marsch, H Schotten, H Tullberg, MA Uusitalo, M Schellmann, in IEEE VTC Spring Workshop, Germany. The foundation of the mobile and wireless communications system for 2020 and beyond (IEEE, Dresden, Germany, 2013), pp. 1-5

137. H Tullberg, P Popovski, D Gozalvez-Serrano, P Fertl, Z Li, A Höglund, MA Uusitalo, H Droste, O Bulakci, J Eichinger, K Pawlak, METIS system concept: the shape of $5 \mathrm{G}$ to come. IEEE Commun Mag (2015). https:// www.metis2020.com/wp-content/uploads/publications/ IEEE_CommMag_2015_Tullberg_etal_METIS-System-Concept.pdf. Accessed 18 August 2015

138. X Jian, X Zeng, Y Jia, L Zhang, Y He, Beta/M/1 Model for machine type communication. EEE Commun. Lett. 17(3), 584-587 (2013)

139. M Iftikhar, B B Landfeldt, S Zeadally, A Zomaya, Service level agreements (SLAs) parameter negotiation between heterogeneous 4G wireless network operators. Pervasive Mob Comput. 7(5), 525-544 (2011)

140. G Latouche, V Ramaswami, A unified stochastic model for the packet stream from periodic sources. Perform. Eval. 14(2), 103-121 (1992)

141. The Internet of Things is poised to change everything, Says IDC. Available from: http://www.businesswire.com/news/home/ 20131003005687/en/Internet-Poised-Change-IDC\#.VXhZkvmqqko_ links.html. Accessed 10 June 2015

142. IEEE 802 16p-11/0014. IEEE 802.16p Machine to Machine (M2M) Evaluation Methodology Document (EMD). IEEE 802.16 Broadband Wireless Access Working Group, (2010)

143. 3GPP. Technical Specification Group Services and System Aspects, Architecture enhancements to facilitate communications with packet data networks and application, 3GPP TS 23.682 V13.1.0, (2012)

144. SC Jha, AT Koc, M Gupta, R Vannithamby, in IEEE First International Black Sea Conference on Communications and Networking (BlackSeaCom). Power saving mechanisms for M2M communication over LTE networks (IEEE, Batumi, Georgia, 2013), pp. 102-106

145. R Ratasuk, A Prasad, Z Li, A Ghosh, M Uusitalo, in IEEE 18th International Conference on Intelligence in Next Generation Networks (ICIN). Recent advancements in M2M communications in $4 \mathrm{G}$ networks and evolution towards 5G, (2015), pp. 52-57

146. oneM2M, Functional architecture. Technical Specification TS-0001-V1.6.1, (2015). Available online: http://www.onem2m.org/ images/files/deliverables/TS-0001-Functional_Architecture-V1_6_1.pdf, accessed 1 June 2015

147. oneM2M, Security Solutions, Technical Specification TS-0003-V1.0.1, (2015). Available online. http://www.onem2m.org/images/files/ deliverables/TS-0003-Security_Solutions-V1_0_1.pdf, accessed 1 June 2015

148. oneM2M, Service layer core protocol specifications. Technical Specification TS-0004-V1.0.1, (2015). Available online: http://www. onem2m.org/technical/published-documents, accessed 1 June 2015
149. oneM2M, CoAP Protocol Binding, Technical Specification TS-0008-V1.0.1, (2015). Available online: http://www.onem2m.org/images/files/ deliverables/TS-0008-CoAP_Protocol_Binding-V1_0_1.pdf, accessed 1 June 2015

150. oneM2M, HTTP Protocol Binding, Technical Specification TS-0009-V1.0.1, (2015). Available online: http://www.onem2m.org/images/files/ deliverables/TS-0009-HTTP_Protocol_Binding-V1_0_1.pdf, accessed 5 March 2015

151. oneM2M, MQTT Protocol Binding, Technical Specification TS-0010-V1.0.1, (2015). Available online: http://www.onem2m.org/ images/files/deliverables/TS-0010-MQTT_protocol_binding-V1_0_1. pdf, accessed 9 March 2015

152. ETSI, Machine-to-Machine Communications (M2M); Study on Semantic support for M2M Data. ETSI TR 101584 V2.1.1, (2013)

153. Mobile and wireless communications Enablers for Twenty-twenty (2020) Information Society, Initial report on horizontal topics, first results and $5 \mathrm{G}$ system concept. (METIS Deliverable Number ICT-317669-METIS/D2.4, 2015). Available online: https://www.metis2020.com/wp-content/ uploads/deliverables/METIS_D2.4_v1.pdf, accessed 15 April 2015

154. Mobile and wireless communications Enablers for Twenty-twenty (2020) Information Society, Proposed solutions for new radio access, (2014). Available online: https://www.metis2020.com/wp-content/uploads/ deliverables/METIS_D6.2_v1.pdf, accessed 15 April 2015

155. EXpAnding LTE for Devices, Impact of use cases in business models, EXALTED Deliverable Number D2.2, (2011). Available online: http:// www.ict-exalted.eu/fileadmin/documents/EXALTED_WP2_D2.2.pdf, accessed 11 November 2014

156. EXpAnding LTE for Devices, First report on LTE-M algorithms and procedures. EXALTED Deliverable Number D3.1, (2011). Available online: http://www.ict-exalted.eu/fileadmin/documents/EXALTED_WP3_D3. 1_v2.0.pdf, accessed 13 November 2014

157. EXpAnding LTE for Devices, First report on LTE-M algorithms and procedures. EXALTED Deliverable Number D3.3, (2012). Available online: http://www.ict-exalted.eu/fileadmin/documents/EXALTED_WP3_D3. 3 v1.0.pdf, accessed 19 November 2014

158. EXpAnding LTE for Devices, The EXALTED system architecture (Final). EXALTED Deliverable Number D2.3, (2012). Available online: http:// www.ict-exalted.eu/fileadmin/documents/EXALTED_WP2_D2.3.pdf, accessed 11 March 2015

159. EXpAnding LTE for Devices, Optimizing a Linux kernel for M2M devices. EXALTED Deliverable Number D6.1, (2011). Available online: http:// www.ict-exalted.eu/fileadmin/documents/EXALTED_WP6_D6.1.pdf, accessed 11 November 2014

160. EXpAnding LTE for Devices, End-to-end (E2E) M2M system-device management. EXALTED Deliverable Number D4.3 (2012). Available online: http://www.ict-exalted.eu/fileadmin/documents/ EXALTED_WP4_D4.3.pdf, accessed 19 December 2014

161. EXALTED, End-to-end (E2E) M2M system-traffic aggregation. Document Deliverable D4.4 (2012)

162. EXALTED, Security and Provisioning Solutions (2012). Deliverable Number D5.1

163. EXALTED, Security Solutions for P2P Relaying (2013). Deliverable Number D5.3

164. LOLA, Specification of PHY/MAC Adaptations for the Target Architectures (2011). Deliverable Number D4.1

165. LOLA, Scheduling Policies for M2M and Gaming Traffic (2011). Deliverable Number D4.5

166. LOLA, Enhancements to Framing and Low-Layer Signalling (2011). Deliverable Number D4.2

167. LOLA, Traffic Models for M2M and Online Gaming Network Traffic (2011). Deliverable Number D3.5

168. EXpAnding LTE for Devices, First Report on Integration of WP3 Traffic Models and WP4 L2 Algorithms on Testbed 1. EXALTED Deliverable Number D3.2, (2012). Available online: http://www.ict-exalted.eu/ fileadmin/documents/EXALTED_WP3_D3.2.pdf. Accessed 26 November 2014

169. C Anton-Haro, M Dohler, Machine-to-Machine (M2M) Communications: Architecture, Performance and Applications. (Woodhead Publishing, Cambridge, UK, 2015). http://store.elsevier.com/product.jsp?isbn= 9781782421023\&pagename=search 
170. 3GPP, Study on provision of low-cost machine-type communications (MTC) user equipments (UEs) based on LTE (Release 12). (3GPP TR 36.888 V12.0.0, 2013)

171. G Velev, M2M applications and 3GPP cellular networks. (NEC Laboratories Europe, 2014). Available online: https://docbox.etsi.org/workshop/2014/ 201412_M2MWORKSHOP/S04_STANDARDS/3GPP_VELEV.pdf, accessed 11 March 2015

172. QUALCOMM, LTEMTC: Optimizing LTE-Advanced for machine-type-communications, (2014). Available online: file:///C:/Users/ yasir/Downloads/lte-mtc-optimizing-Ite-advanced-for-machine-typecommunications\%20(3).pdf, accessed 12 August 2015

Submit your manuscript to a SpringerOpen ${ }^{\circ}$ journal and benefit from:

- Convenient online submission

- Rigorous peer review

- Immediate publication on acceptance

- Open access: articles freely available online

- High visibility within the field

- Retaining the copyright to your article

Submit your next manuscript at $\boldsymbol{\wedge}$ springeropen.com 